\title{
,anc \\ Qubit Regularization and Qubit Embedding Algebras
}

\author{
Hanqing Liu $+(\mathbb{D})$ and Shailesh Chandrasekharan *,t(D) \\ Department of Physics, Duke University, Durham, NC 27708, USA; hanqing.liu@duke.edu \\ * Correspondence: sch27@duke.edu \\ + These authors contributed equally to this work.
}

\begin{abstract}
Qubit regularization is a procedure to regularize the infinite dimensional local Hilbert space of bosonic fields to a finite dimensional one, which is a crucial step when trying to simulate lattice quantum field theories on a quantum computer. When the qubit-regularized lattice quantum fields preserve important symmetries of the original theory, qubit regularization naturally enforces certain algebraic structures on these quantum fields. We introduce the concept of qubit embedding algebras (QEAs) to characterize this algebraic structure associated with a qubit regularization scheme. We show a systematic procedure to derive QEAs for the $\mathrm{O}(N)$ lattice spin models and the $\mathrm{SU}(N)$ lattice gauge theories. While some of the QEAs we find were discovered earlier in the context of the D-theory approach, our method shows that QEAs are far richer. A more complete understanding of the QEAs could be helpful in recovering the fixed points of the desired quantum field theories.
\end{abstract}

Keywords: lattice spin models; lattice gauge theories; quantum computation/simulation; quantum criticality

check for updates

Citation: Liu, H.; Chandrasekharan, S. Qubit Regularization and Qubit Embedding Algebras. Symmetry 2022, 14, 305. https://doi.org/10.3390/ sym14020305

Academic Editors: Simon Hands and Simon Catterall

Received: 15 December 2021 Accepted: 19 January 2022 Published: 2 February 2022

Publisher's Note: MDPI stays neutral with regard to jurisdictional claims in published maps and institutional affiliations.

Copyright: (C) 2022 by the authors. Licensee MDPI, Basel, Switzerland. This article is an open access article distributed under the terms and conditions of the Creative Commons Attribution (CC BY) license (https:// creativecommons.org/licenses/by/ $4.0 /)$.

\section{Introduction}

Recent development in quantum technologies is making the dream of quantum computing into a realistic and exciting possibility [1]. This has triggered the hope that we can overcome the sign problem that plague Monte Carlo calculations of quantum systems [2]. The sign problem arises essentially due to the highly entangled nature of quantum states [3] and currently limits our ability to understand real-time evolution of quantum systems and to compute the ground state properties of fermionic matters. Overcoming these challenges using a quantum computer could help us achieve a much deeper understanding of many fundamental laws of the nature. Quantum simulations of physical systems using simple models [4-13] and both abelian [14-17] and non-abelian [18-23] lattice gauge theories [24] have already been proposed using both analog and digital quantum simulators, such as ultracold atoms in optical lattices [25-31], trapped ions [32-34], and superconducting circuits [35-37].

Simulating quantum field theories using a quantum computer creates its own challenges. Calculations of quantities in quantum field theories are often plagued with infinities and a regularization procedure is necessary to even define the calculation. The word regularization usually refers to a method that removes the ultraviolet infinities that arise due to the presence of an infinite number of quantum degrees of freedom in any "small" spatial region. In the Hamiltonian formulation, a spatial lattice often provides a non-perturbative regularization of these ultraviolet infinities. A quantum critical point in the lattice theory provides a way to remove the lattice artifacts and define the continuum quantum field theory. This is due to the fact that the long distance physics of the model in the vicinity of the correct quantum critical point are described by the renormalization group (RG) fixed point that describes the quantum field theory.

In bosonic quantum field theories, there is yet another type of infinity, i.e., the infinite dimensional Hilbert space at every local lattice site. Local lattice bosonic field $\phi_{\mathbf{r}}$ and its conjugate field $\pi_{\mathbf{r}}$ on spatial sites $\mathbf{r}$ satisfy the canonical commutation relation 


$$
\left[\phi_{\mathbf{r}}, \pi_{\mathbf{r}^{\prime}}\right]=\mathrm{i} \delta_{\mathbf{r}, \mathbf{r}^{\prime}}
$$

which can only be realized with an infinite dimensional local Hilbert space. Although this infinity does not make calculations singular, it does create challenges for simulating quantum field theories on a quantum computer, whose local Hilbert space is finite dimensional. The procedure of regularizing this infinite dimensional local Hilbert space to a finite dimensional one is what we call "qubit regularization", which has been proposed as a first step in simulating quantum field theories using a quantum computer [38-42].

Analogous with ultraviolet regularization, qubit regularization can also be accomplished in many ways. In fact, all lattice quantum spin models that have been studied in condensed matter physics over the years can be considered as examples of qubit regularized models due to their finite dimensional local Hilbert space. Lattice gauge theories were also brought under this framework through the quantum link formulations many years ago [43-45], and quantum simulation based on the quantum link models has also been explored recently [46-49]. A different approach based on discretizing the continuous symmetries has also been proposed [50-53]. Given the limited computational resources in the NISQ era [54], it is crucial to explore the best qubit regularization schemes that can use the limited resources in a clever way. Here, we believe that preserving the symmetries of the original theory should be an important criterion during the regularization procedure, since it may help in constructing qubit Hamiltonians within the basin of attraction of the correct RG fixed point with the same symmetry. In addition, it is also desirable to understand the properties of the regularized Hilbert space and learn if there are interesting hidden mathematical structures that characterize the regularization scheme.

In the qubit-regularized Hilbert space, the bosonic field operators are also regularized to new ones which we can denote as $\phi_{\mathbf{r}}^{Q}$ and $\pi_{\mathbf{r}}^{Q}$ where $Q$ represents some regularization scheme. These qubit-regularized fields generate a unique algebraic structure associated with each qubit regularization scheme, which is referred to as the qubit embedding algebra (QEA). In particular, we are interested in the regularization schemes that preserve the symmetry of the original theory. Therefore, the part of the algebra that guarantees this symmetry cannot change. However, the part of the algebra that is not related to the symmetry is usually not compatible with finite dimensional Hilbert space, and therefore is usually changed. Sacrificing the non-symmetry relations does not necessarily create complications for qubit regularization. From a physical point of view, by preserving the symmetries during qubit regularization at the lattice level, we are guaranteed that long distance physics also preserves them. Since continuum quantum fields arise near fixed points of the RG flow through RG blocking of lattice fields, the continuum operators naturally act on the direct product of an infinite number of local lattice Hilbert spaces. Hence, the relations that are sacrificed at the lattice level due to qubit regularization can be recovered in the continuum limit near the correct quantum critical points.

The idea of QEA was originally developed in the D-theory approach where simple QEAs for various quantum field theories were constructed by hand [45]. In this paper, we develop a systematic way to derive the QEAs starting from the Hilbert space of the traditional theory, which can be viewed as a direct sum of many irreducible representations (irreps) of the symmetry group [55-58]. We define each qubit regularization scheme as a projection operator $P_{Q}$, which projects the original infinite dimensional space to some finite set of irreps $Q$. This projection naturally preserves important algebra related to the symmetries of the original theory. Furthermore, it also naturally defines a QEA that depends on $Q$. In this work, we explicitly construct simple QEAs for $O(N)$ lattice spin models and $\mathrm{SU}(N)$ lattice gauge theories. Some of these are the same as the ones discovered earlier in the D-theory context, but we also find new ones.

Qubit regularization is only the first step in the process of studying quantum field theories using a quantum computer. The second step is the construction of qubit Hamiltonians that contain the correct quantum critical point where the desired continuum quantum 
field theory emerges. Finally, one must understand which of the viable Hamiltonians lead to an efficient implementation of the associated quantum circuits. In this work, we only focus only on the first step and leave several questions for future research. Can all desirable quantum field theories be constructed using a fixed finite dimensional local Hilbert space? The answer to this question is not clear and needs further research. There is strong evidence that conformal field theories that emerge at Wilson-Fisher fixed points can be recovered using fixed finite dimensional local Hilbert spaces $[39,59,60]$. We have also recently discovered that some asymptotically free theories can also be recovered in this way [41]. In fact, D-theory suggests that one can recover many traditional lattice theories with infinite dimensional Hilbert spaces within acceptable errors, by using simple qubit regularized models. In addition, the errors shrink exponentially fast as the size of the local Hilbert space increases $[42,45,61]$.

Which qubit regularized model leads to the most efficient implementation on a quantum computer? This is an important question to address in the noisy intermediate-scale quantum (NISQ) era, when large reliable quantum computers will not be available. For example, in the context of gauge theories, can we reduce the Hilbert space dramatically by implementing the Gauss law constraint $[62,63]$ ? However, before we can compare various qubit regularized models, we first need to identify which models are worth comparing by searching for those that contain the correct quantum critical point. If we can solve the qubit regularized models directly by other methods in some parameter range, we may be able identify those that have the correct physics. Unfortunately, the method of perturbation theory that exists with traditional regularization schemes is not easily available for qubit regularized models. Furthermore, qubit regularized models can suffer from sign problems even though traditional models are free of them. Hence, classical Monte Carlo methods are also not easily available to study qubit regularized models. On the other hand, when sign problems are absent, quantum Monte Carlo methods similar to the ones used to solve traditional models can also be designed for qubit regularized models. Such a method led to the discovery of a two-qubit model that reproduces the asymptotically free two-dimensional $\mathrm{O}$ (3) model [41]. Methods based on tensor networks also seem promising and currently being developed for a variety of lattice field theories [24,64].

Our paper is organized as follows: In Section 2, we develop a systematic approach to qubit-regularize the $\mathrm{O}(N)$ nonlinear sigma model and show how the QEAs arise. We fully characterize the QEAs of the $\mathrm{O}(2)$ model in Section 2.1 and calculate several simple examples of QEAs for the $\mathrm{O}(3)$ model in Section 2.2. In Section 2.3, we extend the analysis of the $\mathrm{O}(3)$ case to $\mathrm{O}(N)$ and show the existence of a simple QEA for any N. In Section 3, we show how to extend our systematic approach to qubit-regularize $S U(N)$ gauge theories. We derive the QEA in the simplest regularization schemes for the SU(2) lattice gauge theory in Section 3.1, for the SU(3) lattice gauge theory in Section 3.2, and generalize these analyses to the $S U(N)$ lattice gauge theory and discuss the physical meaning of each element in the QEA in Section 3.3. Some other regularization schemes for SU(3) obtained with a mathematical software package called GAP [65] are also discussed in Section 3.2. Finally, in Section 4, we discuss the nice features of our simple regularization scheme in $\mathrm{SU}(N)$ gauge theories and present some conjectures about QEAs that could emerge for any $N$.

\section{Lattice Spin Models}

In order to understand the algebraic structure that qubit regularization imposes on the Hilbert space of lattice field theories, it is instructive to begin with lattice spin models. Let us consider $\mathrm{O}(N)$ invariant nonlinear sigma models in the Hamiltonian formalism on a lattice. These can be described through $\mathrm{O}(N)$ quantum rotor models whose Hamiltonian is given by

$$
H=\frac{1}{2 \beta} \sum_{\mathbf{r}, a} L_{\mathbf{r}}^{a} L_{\mathbf{r}}^{a}-\beta \sum_{\left\langle\mathbf{r}, \mathbf{r}^{\prime}\right\rangle, i} \phi_{\mathbf{r}}^{i} \phi_{\mathbf{r}^{\prime}}^{i},
$$


where $\mathbf{r}$ labels the spatial lattice sites and $\langle\cdot, \cdot\rangle$ refers to nearest neighbors. Here, $L_{\mathbf{r}}^{a}$ $a=1,2, \cdots, N(N-1) / 2$ are the generators of the $\mathrm{O}(N)$ rotations, while $\phi_{\mathbf{r}}^{i}, i=1,2, \cdots, N$ transform in the fundamental representation of $\mathrm{O}(N)$, i.e.,

$$
\left[L_{\mathbf{r}}^{a}, L_{\mathbf{r}^{\prime}}^{b}\right]=\mathrm{i} f^{a b c} L_{\mathbf{r}}^{c} \delta_{\mathbf{r}, \mathbf{r}^{\prime},}\left[L_{\mathbf{r}}^{a}, \phi_{\mathbf{r}^{\prime}}^{i}\right]=\mathrm{i}\left(T^{a}\right)_{j}^{i} \phi_{\mathbf{r}}^{j} \delta_{\mathbf{r}, \mathbf{r}^{\prime},}
$$

where $T^{a}$ is the fundamental representation of $L^{a}$, and $f^{a b c}$ are structure constants of the $\mathrm{O}(N)$ algebra. Since Equation (3) is dictated by the symmetry of the theory and specifies how the fields transform under the symmetry, we will refer to an algebra of this kind as an extended symmetry algebra.

In the traditional lattice nonlinear sigma models, $\phi_{\mathbf{r}}^{i}$ are usually the position operators on a certain homogeneous target space associated with a site $\mathbf{r}, L_{\mathbf{r}}^{a}$ generate translations on this target space and therefore can be viewed as momentum operators (more precisely, when projected to the tangent spaces of the target space, $L_{\mathbf{r}}^{a}$ give the momentum operators), and the local Hilbert space is the space of square integrable functions on this target space. In our case, the target space is an $N-1$ dimensional sphere $S^{N-1} \cong \mathrm{O}(N) / \mathrm{O}(N-1)$, and the Hilbert space $\mathcal{H}$ is $L^{2}\left(S^{N-1}\right)$. Notice that the position eigenstates simultaneously diagonalize the $\phi_{\mathbf{r}}^{i}$ operators, and we have the following relations:

$$
\left[\phi_{\mathbf{r}}^{i}, \phi_{\mathbf{r}^{\prime}}^{j}\right]=0, \quad \sum_{i} \phi_{\mathbf{r}}^{i} \phi_{\mathbf{r}}^{i}=1
$$

Since the goal here is to recover $\mathrm{O}(N)$ invariant QFTs in the continuum limit, the exact form of the Hamiltonian in Equation (2) is not important as long as it can be tuned to the correct quantum critical point where the QFT emerges. In order to accomplish this, it is important to preserve the symmetries of the theory, which in our case is the $\mathrm{O}(N)$ symmetry. Therefore, it is important to preserve the extended symmetry algebras Equation (3). On the other hand, the relations in Equation (4) are not necessary from the perspective of symmetry. It turns out that Equations (3) and (4) can only be realized via an infinite dimensional local Hilbert space. Therefore, Equation (4) has to be sacrificed in order to have a finite dimensional local Hilbert space. Since QEA is a local concept, it is independent of the site $\mathbf{r}$. For this reason, in our discussions below, we will suppress this spatial index $\mathbf{r}$ in the operators $L_{\mathbf{r}}^{a}$ and $\phi_{\mathbf{r}}^{i}$ and simply refer to them as $L^{a}$ and $\phi^{i}$.

One systematic way to "qubit-regularize" the Hilbert space to a finite dimensional one, while preserving Equation (3) is through working with the "momentum eigen-blocks" of the Hilbert space $L^{2}\left(S^{N-1}\right)$, i.e., decomposing $L^{2}\left(S^{N-1}\right)$ into irreps of the symmetry algebra $\mathrm{O}(N)$. From the standard results of harmonic analysis on spheres, we know that

$$
L^{2}\left(S^{N-1}\right)=\bigoplus_{l=0}^{\infty} V_{l},
$$

where $V_{l}$ is the space of spherical harmonics of degree $l$ which are the momentum eigenblocks. Furthermore, each $V_{l}$ forms an irrep of $\mathrm{O}(N)$ with $\operatorname{dim} V_{l}=\left(\begin{array}{c}N+l-1 \\ l\end{array}\right)-\left(\begin{array}{c}N+l-3 \\ l-2\end{array}\right)$, which means the operators $L^{a}$ act within each momentum eigenblock. On the other hand, $\phi^{i}$ behave like raising and lowering operators and connect different eigen-blocks. Then, we can choose a finite set of integers denoted by $Q$, and qubit-regularize the Hilbert space to

$$
\mathcal{H}_{Q}:=\bigoplus_{l \in Q} V_{l}
$$

We can define a projector to this space by

$$
P_{Q}:=\sum_{l \in Q} P_{l}
$$


where $P_{l}$ is a projector to $V_{l}$. Then, the operators in the truncated theory is simply defined to be the original operators projected to $\mathcal{H}_{Q}$, i.e., $L_{Q}^{a}:=P_{Q} L^{a} P_{Q}$ and $\phi_{Q}^{i}:=P_{Q} \phi^{i} P_{Q}$. Since $\left[L^{a}, P_{Q}\right]=0$, it is easy to verify that

$$
\left[L_{Q^{a}}^{a} L_{Q}^{b}\right]=\mathrm{i} f^{a b c} L_{Q^{c}}^{c}, \quad\left[L_{Q^{\prime}}^{a} \phi_{Q}^{i}\right]=\mathrm{i}\left(T^{a}\right)_{j}^{i} \phi_{Q^{\prime}}^{j}
$$

i.e., the extended symmetry algebra Equation (3) is preserved. Since $\phi^{i}$ are raising and lowering operators between different $l^{\prime}$ s and $Q$ is a finite set, $\left[P_{Q}, \phi^{i}\right] \neq 0$ and thus $\left[\phi_{Q^{\prime}}^{i} \phi_{Q}^{j}\right]$ need not vanish. We will show that $L_{Q^{\prime}}^{a} \phi_{Q}^{i}$ generate a QEA that depends on the choice of $Q$. To illustrate this idea more concretely, we will work out explicit examples in some simple cases below and then extend the idea to lattice gauge theories in the next section.

\subsection{O(2) Lattice Spin Model}

We begin with the simplest example of the $\mathrm{O}(2)$ spin model on the lattice which has been considered earlier in $[17,66]$, but, as far as we can tell, the idea of QEA was not fully explored. The traditional lattice theory is constructed using three operators, the angular momentum operator $L$ that generates $\mathrm{O}(2)$ transformations and the fields $\phi^{1}$ and $\phi^{2}$. The extended symmetry algebra that replaces Equation (3) is now given by

$$
\left[L, \phi^{ \pm}\right]= \pm \phi^{ \pm},
$$

where we define $\phi^{ \pm}=\frac{1}{\sqrt{2}}\left(\phi^{1} \pm \mathrm{i} \phi^{2}\right)$. In the nonlinear realization of the traditional lattice theory, we further impose the relations

$$
\left[\phi^{1}, \phi^{2}\right]=0, \quad\left(\phi^{1}\right)^{2}+\left(\phi^{2}\right)^{2}=1,
$$

which are not required from the symmetry perspective. These extra relations force the Hilbert space at each lattice site to be infinite dimensional.

The idea of qubit regularization of the $\mathrm{O}(2)$ model is to define three new operators $L_{Q}, \phi_{Q}^{1}$ and $\phi_{Q}^{2}$, which act on a finite dimensional local Hilbert space, satisfy the extended symmetry algebra Equation (9), but replace the extra relations Equation (10) with something else. To accomplish this systematically, we first construct the orthonormal "position" basis that are eigenstates of the field operators $\phi^{1}$ and $\phi^{2}$. These are given by $|\theta\rangle, 0 \leq \theta<2 \pi$ and satisfy

$$
\left\langle\theta \mid \theta^{\prime}\right\rangle=\delta\left(\theta-\theta^{\prime}\right), \quad \int_{0}^{2 \pi} \mathrm{d} \theta|\theta\rangle\langle\theta|=1 .
$$

In this basis, $\phi^{1}$ and $\phi^{2}$ are diagonal, $\phi^{1}|\theta\rangle=\cos \theta|\theta\rangle, \phi^{2}|\theta\rangle=\sin \theta|\theta\rangle$, but the "angular momentum" operator $L$ is not. In order to construct the qubit regularized fields $L_{Q}, \phi_{Q}^{1}$ and $\phi_{Q}^{2}$, we have to work in the basis that naturally expresses the Hilbert space as a direct sum of irreps of the $\mathrm{O}(2)$ symmetry. These are eigenstates of the angular momentum operator, given by $|k\rangle, k=0, \pm 1, \pm 2, \cdots$. They are related to the position eigenstates $|\theta\rangle$ through the relation

$$
\langle\theta \mid k\rangle=\frac{1}{\sqrt{2 \pi}} \mathrm{e}^{\mathrm{i} k \theta}
$$

i.e., $|k\rangle$ is related to $|\theta\rangle$ via Fourier transform. As we will see later, in all the $\mathrm{O}(N)$ models and $\mathrm{SU}(N)$ gauge theories we are going to discuss, the relations between "position" eigenstates and "momentum" eigenblocks can be viewed as certain generalizations of the Fourier transform. While $L$ is diagonal in the angular momentum basis $L|k\rangle=k|k\rangle$, 
the field operators $\phi^{1}$ and $\phi^{2}$ are not. In fact, $\phi^{ \pm}$act as raising and lowering operators of angular momenta

$$
\phi^{ \pm}|k\rangle=\frac{1}{\sqrt{2}}|k \pm 1\rangle
$$

for all values of $k$.

We can now qubit-regularize our theory by projecting the traditional Hilbert space to a finite dimensional subspace using the projector

$$
P_{Q}=\sum_{k \in Q}|k\rangle\langle k|,
$$

where $Q$ is a set of allowed irreps of the $\mathrm{O}(2)$ symmetry. We can then define $L_{Q}=P_{Q} L P_{Q}$, $\phi_{Q}^{ \pm}=P_{Q} \phi^{ \pm} P_{Q}$ as the qubit regularized fields. One simple choice is $Q=\left\{k \mid k_{\min } \leq\right.$ $\left.k \leq k_{\max }\right\}$, which is a single block of consecutive integers. This implies $\phi_{Q}^{+}\left|k_{\max }\right\rangle=0$ and $\phi_{Q}^{-}\left|k_{\min }\right\rangle=0$. In this regularization scheme, the original infinite dimensional representation is replaced by a $d=k_{\max }-k_{\min }+1$ dimensional Hilbert space. It is easy to verify that the operators $L_{Q}$ and $\phi_{O}^{ \pm}$are now $d \times d$ matrices that satisfy the extended symmetry algebra Equation (9), but not Equation (10). We can construct the operators explicitly by computing the matrix elements:

$$
\left(L_{Q}\right)_{k k^{\prime}}=\left\langle k|L| k^{\prime}\right\rangle=k \delta_{k, k^{\prime},} \quad\left(\phi_{Q}^{ \pm}\right)_{k k^{\prime}}=\left\langle k\left|\phi^{ \pm}\right| k^{\prime}\right\rangle=\frac{1}{\sqrt{2}} \delta_{k, k^{\prime} \pm 1}
$$

where $k_{\min } \leq k, k^{\prime} \leq k_{\max }$. Starting from this matrix representation of $L_{Q}$ and $\phi_{Q}^{ \pm}$, we can construct linear combinations of nested commutators until no new matrices are generated. They form a closed Lie algebra, which is what we called the QEA. We note that in the definition of an algebra of commutators, one usually chooses to work with traceless matrices. In our case, the only matrix that may not be traceless is $L_{Q}$. We can always shift $L_{Q}$ by a proper multiple of identity to make it traceless. As we prove in Appendix A, the QEA for $Q=\left\{k \mid k_{\min } \leq k \leq k_{\max }\right\}$ is the Lie algebra of the $\mathrm{SO}(d)$ or $\mathrm{Sp}(d / 2)$ when $d$ is odd or even, respectively.

What is the QEA if we choose $Q$ to be a set of consecutive integer blocks that are themselves not consecutive, for example, $Q=\{1,2,3,5,6,7,8\}$ ? This question is perhaps not so interesting since it is unlikely one will choose such a complicated qubit regularization scheme, but it is a valid mathematical question. Since $\phi^{ \pm}$only connect consecutive integer blocks, $L_{Q}$ and $\phi_{Q}^{ \pm}$are block diagonal. We already know when projected to each consecutive integer block in $Q$, the QEA is either $\mathrm{SO}(d)$ or $\mathrm{Sp}(d / 2)$, but the full QEA is not necessarily a direct product of $\mathrm{SO}(d)$ and $\mathrm{Sp}(d / 2)$ because the components can be possibly correlated. From Goursat's lemma [67], we know that two simple Lie groups cannot be correlated unless they share the same Lie algebra. In our case, both $\mathrm{SO}(d)$ for $d$ odd and $\mathrm{Sp}(d / 2)$ for $d$ even are simple Lie groups, and the Lie groups with different $d$ 's have different Lie algebras, except for the accidental isomorphisms $\mathfrak{s o}(3) \cong \mathfrak{s p}(1)$ and $\mathfrak{s o}(5) \cong \mathfrak{s p}(2)$, whose possible correlations can be easily ruled out numerically using a mathematical package called GAP [65]. Therefore, we conclude that any two blocks cannot be correlated when they have different dimensions $d$. What happens when two blocks have the same dimension $d$ ? Actually, in this case, the two blocks are fully correlated because up to a shift by identity, the generators $L$ and $\phi^{ \pm}$are always identical in the two sectors, and therefore the commutators are also always identical. Therefore, we know two blocks are independent if they have different dimensions, and they are fully correlated otherwise. However, can there be any correlation among multiple blocks, even though they are pairwise independent? This possibility can be ruled out using Serre's Lemma [68], which states that pairwise independence implies full independence for perfect groups, which include $\mathrm{SO}(d)$ and $\mathrm{Sp}(d / 2)$. In summary, if the distinct dimensions of the consecutive integer blocks in $Q$ are $d_{1}, d_{2}, \cdots, d_{m}$, where "distinct" means that if two $d^{\prime}$ s are equal, we only count them 
once, then the QEA is $\mathrm{SO}\left(d_{1}\right) \times \mathrm{SO}\left(d_{2}\right) \times \cdots \times \mathrm{SO}\left(d_{m}\right)$ assuming all $d_{i}$ 's are odd. If any one of the $d_{i}$ is even, we just replace the corresponding block with $\operatorname{Sp}\left(d_{i} / 2\right)$. In the example $Q=\{1,2,3,5,6,7,8\}$, the QEA would be $\mathrm{SO}(3) \times \mathrm{Sp}(2)$. From this example, we see clearly that the QEA will depend on the choice of the regularization scheme $Q$.

\section{2. $\mathrm{O}(3)$ Lattice Spin Model}

Let us extend the above analysis to the traditional lattice $\mathrm{O}(3)$ spin model and explore the corresponding QEAs. In this case, the lattice model is constructed with quantum fields $L^{a}, a=1,2,3$ which are the generators of the $\mathrm{O}(3)$ group, and $\phi^{i}, i=1,2,3$ which transform as a vector under $\mathrm{O}(3)$. The extended symmetry algebra is now given by

$$
\left[L^{a}, L^{b}\right]=\mathrm{i} \varepsilon^{a b c} L^{c}, \quad\left[L^{a}, \phi^{i}\right]=\mathrm{i} \varepsilon^{a i j} \phi^{j} .
$$

In the traditional lattice model, we impose the extra constraints

$$
\left[\phi^{i}, \phi^{j}\right]=0, \quad \sum_{i}\left(\phi^{i}\right)^{2}=1,
$$

which are not necessary from the symmetry perspective. The local Hilbert space that realizes these relations is the space of all square integrable functions on a sphere, denoted by $L^{2}\left(S^{2}\right)$, which is infinite dimensional.

In order to qubit-regularize the $\mathrm{O}(3)$ model, we will need to construct operators $L_{Q}^{a}$ and $\phi_{Q}^{i}$ that act on a finite dimensional local Hilbert space, satisfying the extended symmetry algebra Equation (16). To accomplish this, we first construct the orthonormal "position" basis that are eigenstates of $\phi^{i}$. These are given by $|\theta, \varphi\rangle, 0 \leq \theta \leq \pi, 0 \leq \varphi<2 \pi$, where $\theta$ and $\varphi$ are the spherical coordinates. The position basis states satisfy

$$
\left\langle\theta, \varphi \mid \theta^{\prime}, \varphi^{\prime}\right\rangle=\frac{1}{\sin \theta} \delta\left(\theta-\theta^{\prime}\right) \delta\left(\varphi-\varphi^{\prime}\right), \quad \int \mathrm{d} \Omega|\theta, \varphi\rangle\langle\theta, \varphi|=1 .
$$

In this basis, $\phi^{i}$ are diagonal,

$$
\phi^{1}|\theta, \varphi\rangle=\sin \theta \cos \varphi|\theta, \varphi\rangle, \quad \phi^{2}|\theta, \varphi\rangle=\sin \theta \sin \varphi|\theta, \varphi\rangle, \quad \phi^{3}|\theta, \varphi\rangle=\cos \theta|\theta, \varphi\rangle,
$$

but not the "angular momentum" operators $L^{a}$. In order to construct $L_{Q}^{a}$ and $\phi_{Q^{\prime}}^{i}$ we work in the basis that expresses the original Hilbert space as a direct sum of irreps of the $\mathrm{O}(3)$ symmetry group. These are the angular momentum eigenstates labeled by $|\ell, m\rangle$, $\ell=0,1,2, \cdots$ and $-\ell \leq m \leq \ell$. They form a complete orthonormal basis,

$$
\left\langle\ell, m \mid \ell^{\prime}, m^{\prime}\right\rangle=\delta_{\ell \ell^{\prime}} \delta_{m m^{\prime}}, \quad \sum_{\ell=0}^{\infty} \sum_{m=-\ell}^{\ell}|\ell, m\rangle\langle\ell, m|=1 .
$$

and can be related to the position basis through the relation

$$
\langle\theta, \varphi \mid \ell, m\rangle=Y_{\ell}^{m}(\theta, \varphi),
$$

where $Y_{\ell}^{m}(\theta, \varphi)$ are the usual spherical harmonics. While $L^{a}$ are block diagonal in the angular momentum basis, $\phi^{i}$ are vector operators that mix $\ell$ with $\ell \pm 1$. To understand how, it is convenient to combine $\phi^{1}$ and $\phi^{2}$ into raising and lowering operators $\phi^{ \pm}=\frac{1}{\sqrt{2}}\left(\phi^{1} \pm \mathrm{i} \phi^{2}\right)$. Using the Wigner-Eckart theorem, we can show that

$$
\begin{aligned}
\mp \phi^{ \pm}|\ell, m\rangle & =r_{\ell+1, \ell} c_{1}^{ \pm}|\ell+1, m \pm 1\rangle+r_{\ell, \ell} c_{0}^{ \pm}|\ell, m \pm 1\rangle+r_{\ell-1, \ell} c_{-1}^{ \pm}|\ell-1, m \pm 1\rangle, \\
\phi^{3}|\ell, m\rangle & =r_{\ell+1, \ell} c_{1}^{3}|\ell+1, m\rangle+r_{\ell, \ell} c_{0}^{3}|\ell, m\rangle+r_{\ell-1, \ell} c_{-1}^{3}|\ell-1, m\rangle,
\end{aligned}
$$


where $r_{\ell, \ell^{\prime}}$ are the reduced matrix elements given by

$$
r_{\ell+1, \ell}=\sqrt{\frac{\ell+1}{2 \ell+3}}, \quad r_{\ell, \ell}=0, \quad r_{\ell-1, \ell}=-\sqrt{\frac{\ell}{2 \ell-1}},
$$

and $c_{q}^{ \pm}, c_{q}^{3}, q=0, \pm 1$ are the Clebsch-Gordan coefficients,

$$
\begin{aligned}
c_{1}^{ \pm} & =\sqrt{\frac{(\ell \pm m+1)(\ell \pm m+2)}{(2 \ell+1)(2 \ell+2)}}, \quad c_{1}^{3}=\sqrt{\frac{(\ell-m+1)(\ell+m+1)}{(2 \ell+1)(\ell+1)}}, \\
c_{0}^{ \pm} & =\mp \sqrt{\frac{(\ell \mp m)(\ell \pm m+1)}{2 \ell(\ell+1)}}, \quad c_{0}^{3}=\frac{m}{\sqrt{\ell(\ell+1)}}, \\
c_{-1}^{ \pm} & =\sqrt{\frac{(\ell \mp m-1)(\ell \mp m)}{2 \ell(2 \ell+1)}}, \quad c_{-1}^{3}=-\sqrt{\frac{(\ell-m)(\ell+m)}{(2 \ell+1) \ell}} .
\end{aligned}
$$

Using Equation (22), we can compute all matrix elements of $\phi^{ \pm}$and $\phi^{3}$ between angular momentum basis states.

Analogous with the $\mathrm{O}(2)$ case, we define $P_{Q}$ as a projector into a subspace of allowed values of $\ell^{\prime}$ s,

$$
P_{Q}=\sum_{\ell \in Q} \sum_{m=-\ell}^{\ell}|\ell, m\rangle\langle\ell, m| .
$$

Using $P_{Q}$, we can define the qubit regularized fields $L_{Q}^{a}=P_{Q} L^{a} P_{Q}$ and $\phi_{Q}^{i}=P_{Q} \phi^{i} P_{Q}$. These fields satisfy the extended symmetry algebra Equation (16), but not Equation (17). We can construct them explicitly as matrices once we know $\left\langle\ell^{\prime}, m^{\prime}\left|L^{a}\right| \ell, m\right\rangle$ and $\left\langle\ell^{\prime}, m^{\prime}\left|\phi^{i}\right| \ell, m\right\rangle$. The former is simply the spin- $\ell$ representation of angular momentum operators, and we just learned how to compute the latter. Let us consider two simple examples below. we have

As a first example, we choose $Q=\{0,1\}$. Then, in the basis $(|1,1\rangle,|1,0\rangle,|1,-1\rangle,|0,0\rangle)^{T}$,

$$
L_{Q}^{1}=\frac{1}{\sqrt{2}}\left(\begin{array}{llll}
0 & 1 & 0 & 0 \\
1 & 0 & 1 & 0 \\
0 & 1 & 0 & 0 \\
0 & 0 & 0 & 0
\end{array}\right), \quad L_{Q}^{2}=\frac{1}{\sqrt{2}}\left(\begin{array}{cccc}
0 & -\mathrm{i} & 0 & 0 \\
\mathrm{i} & 0 & -\mathrm{i} & 0 \\
0 & \mathrm{i} & 0 & 0 \\
0 & 0 & 0 & 0
\end{array}\right), \quad L_{Q}^{3}=\left(\begin{array}{cccc}
1 & 0 & 0 & 0 \\
0 & 0 & 0 & 0 \\
0 & 0 & -1 & 0 \\
0 & 0 & 0 & 0
\end{array}\right) .
$$

and

$$
\phi_{Q}^{1}=\frac{1}{\sqrt{6}}\left(\begin{array}{cccc}
0 & 0 & 0 & -1 \\
0 & 0 & 0 & 0 \\
0 & 0 & 0 & 1 \\
-1 & 0 & 1 & 0
\end{array}\right), \quad \phi_{Q}^{2}=\frac{1}{\sqrt{6}}\left(\begin{array}{cccc}
0 & 0 & 0 & \mathrm{i} \\
0 & 0 & 0 & 0 \\
0 & 0 & 0 & \mathrm{i} \\
-\mathrm{i} & 0 & -\mathrm{i} & 0
\end{array}\right), \quad \phi_{Q}^{3}=\frac{1}{\sqrt{3}}\left(\begin{array}{cccc}
0 & 0 & 0 & 0 \\
0 & 0 & 0 & 1 \\
0 & 0 & 0 & 0 \\
0 & 1 & 0 & 0
\end{array}\right) .
$$

The commutators between the six operators $L_{Q}^{a}$ and $\phi_{Q}^{i}$ can be calculated explicitly,

$$
\left[L_{Q^{\prime}}^{a} L_{Q}^{b}\right]=\mathrm{i} \varepsilon^{a b c} L_{Q^{\prime}}^{c}, \quad\left[L_{Q^{\prime}}^{a} \phi_{Q}^{i}\right]=\mathrm{i} \varepsilon^{a i j} \phi_{Q^{\prime}}^{j} \quad\left[\phi_{Q^{\prime}}^{i} \phi_{Q}^{j}\right]=\frac{1}{3} \mathrm{i} \varepsilon^{i j a} L_{Q^{\prime}}^{a}
$$

which is a closed Lie algebra. We can define $J_{ \pm}^{a}=L_{Q}^{a} \pm \sqrt{3} \phi_{Q^{\prime}}^{a}$ and then $J_{+}^{a}$ and $J_{-}^{a}$ form the Lie algebra of two commuting $\mathrm{SU}(2)$, which is isomorphic to the Lie algebra of $\mathrm{SO}(4)$. Thus, we learn that the QEA is the Lie algebra of $\mathrm{SO}(4)$ when $Q=\{0,1\}$.

A simpler qubit regularization scheme to choose would have been $Q=\{\ell\}$, i.e., a single irrep of $\mathrm{O}(3)$. From Equation (23), we notice that $r_{\ell, \ell}=0$, which means $\phi_{Q}^{i}=0$ in this case. Then, the QEA is simply the Lie algebra of $\mathrm{SO}(3)$. Qubit models constructed within this regularization scheme will simply be quantum spin- $\ell$ models. Some may find it disturbing that $\phi^{i}$ has disappeared in this approach and so may feel that we have changed 
the physics in some fundamental way through this regularization scheme. However, in the continuum limit, these fields can still emerge. To see this, it is important to remember that we are only discussing the Hilbert space in the ultraviolet and the full Hilbert of the lattice theory is much richer. By constructing an appropriate qubit Hamiltonian, we can recover all the relevant fields of the original $\mathrm{O}(3)$ quantum field theory at long distances through renormalization group blocking. It is well known that we can recover the physics of the Wilson-Fisher fixed point at the order-disorder quantum phase transition using an appropriate quantum spin- $\ell$ Hamiltonian [59].

\section{3. $\mathrm{O}(N)$ Lattice Spin Model}

We can extend the analysis of the previous two subsections to general $\mathrm{O}(N)$ spin models, although a complete discussion can become progressively more complex. In this section, we prove a simple result regarding the QEA that one obtains if we choose $Q=\{0,1\}$ in Equation (6), i.e., the trivial and fundamental representation. From the previous two subsections, we observe that, when $N=2,3$, for this choice of $Q$, the QEA is $\mathrm{SO}(N+1)$. This pattern is actually true for all $N \geq 2$. Let us now give a brief argument for this result.

The traditional Hilbert space of $\mathrm{O}(N)$ models is given by square integrable functions on the $(N-1)$-sphere $L^{2}\left(S^{N-1}\right)$. The orthonormal "position" basis in this case is labeled by $N-1$ angles $\left|\theta_{1}, \theta_{2}, \cdots, \theta_{N-1}\right\rangle$ where $0 \leq \theta_{i} \leq \pi$ for $1 \leq i \leq N-2,0 \leq \theta_{N-1}<2 \pi$. They are eigenstates of $\phi^{i}$, with eigenvalues

$$
\begin{aligned}
\phi^{1}\left|\theta_{1}, \theta_{2}, \cdots, \theta_{N-1}\right\rangle & =\cos \theta_{1}\left|\theta_{1}, \theta_{2}, \cdots, \theta_{N-1}\right\rangle \\
\phi^{2}\left|\theta_{1}, \theta_{2}, \cdots, \theta_{N-1}\right\rangle & =\sin \theta_{1} \cos \theta_{2}\left|\theta_{1}, \theta_{2}, \cdots, \theta_{N-1}\right\rangle \\
& \vdots \\
\phi^{N-1}\left|\theta_{1}, \theta_{2}, \cdots, \theta_{N-1}\right\rangle & =\sin \theta_{1} \cdots \sin \theta_{N-2} \cos \theta_{N-1}\left|\theta_{1}, \theta_{2}, \cdots, \theta_{N-1}\right\rangle \\
\phi^{N}\left|\theta_{1}, \theta_{2}, \cdots, \theta_{N-1}\right\rangle & =\sin \theta_{1} \cdots \sin \theta_{N-2} \sin \theta_{N-1}\left|\theta_{1}, \theta_{2}, \cdots, \theta_{N-1}\right\rangle .
\end{aligned}
$$

Generalizing the concept of the solid angle, we have the following uniform measure on $S^{N-1}$ parameterized by the $N-1$ angles,

$$
\mathrm{d} \Omega_{N-1}=\mathrm{d} \theta_{1} \mathrm{~d} \theta_{2} \cdots \mathrm{d} \theta_{N-1} \sin ^{N-2} \theta_{1} \sin ^{N-3} \theta_{2} \cdots \sin \theta_{N-2}
$$

such that

$$
\begin{gathered}
\left\langle\theta_{1}, \cdots, \theta_{N-1} \mid \theta_{1}^{\prime}, \cdots, \theta_{N-1}^{\prime}\right\rangle=\frac{1}{\sin ^{N-2} \theta_{1} \cdots \sin \theta_{N-2}} \delta\left(\theta_{1}-\theta_{1}^{\prime}\right) \cdots \delta\left(\theta_{N-1}-\theta_{N-1}^{\prime}\right), \\
\int \mathrm{d} \Omega_{N-1}\left|\theta_{1}, \theta_{2}, \cdots, \theta_{N-1}\right\rangle\left\langle\theta_{1}, \theta_{2}, \cdots, \theta_{N-1}\right|=1 .
\end{gathered}
$$

The surface area of $S^{N-1}$ is given by $\Omega_{N-1}=\frac{2 \pi^{N / 2}}{\Gamma(N / 2)}$.

As before, the "angular momentum" basis states are simply the irreps of the $\mathrm{O}(N)$ symmetry, and $L^{2}\left(S^{N-1}\right)$ is a direct sum of these irreps given in Equation (5). We know from the standard results from harmonics analysis on spheres that there is a one-to-one correspondence between $V_{l}$ and degree $l$ homogeneous polynomials in $\mathbb{R}^{N}$ which are solutions to the Laplacian operator $\nabla^{2}$. More concretely, $V_{0}$ is simply the space of constant functions over the sphere spanned by a single state which we label as $|0\rangle$, while $V_{1}$ is an $N$ dimensional vector space spanned by the coordinate functions on $S^{N-1}$. An orthonormal 
basis of $V_{1}$ can be labeled as $|i\rangle, i=1,2, \cdots, N$, and are related to the "position" eigenstates through the following relations:

$$
\begin{aligned}
\left\langle\theta_{1}, \theta_{2}, \cdots, \theta_{N-1} \mid 0\right\rangle & =\sqrt{1 / \Omega_{N-1}} \\
\left\langle\theta_{1}, \theta_{2}, \cdots, \theta_{N-1} \mid 1\right\rangle & =\sqrt{N / \Omega_{N-1}} \cos \theta_{1} \\
& \vdots \\
\left\langle\theta_{1}, \theta_{2}, \cdots, \theta_{N-1} \mid N-1\right\rangle & =\sqrt{N / \Omega_{N-1}} \sin \theta_{1} \cdots \sin \theta_{N-2} \cos \theta_{N-1} \\
\left\langle\theta_{1}, \theta_{2}, \cdots, \theta_{N-1} \mid N\right\rangle & =\sqrt{N / \Omega_{N-1}} \sin \theta_{1} \cdots \sin \theta_{N-2} \sin \theta_{N-1} .
\end{aligned}
$$

These are eigenstates of $L^{a}$, but not $\phi^{i}$. Using these results, we can show that

$$
\left\langle m\left|\phi^{i}\right| n\right\rangle=\frac{1}{\sqrt{N}}\left(\delta_{m, 0} \delta_{n, i}+\delta_{m, i} \delta_{n, 0}\right) .
$$

In the basis of Equation (32), we can also compute the matrix elements of $L^{a}$, and it is convenient to relabel the $\frac{1}{2} N(N-1)$ indices $a$ in terms of indices $i j$ where $1 \leq i<j \leq N$,

$$
\left\langle m\left|L^{i j}\right| n\right\rangle=-\mathrm{i}\left(\delta_{m, i} \delta_{n, j}-\delta_{m, j} \delta_{n, i}\right) .
$$

Using Equations (33) and (34), we can compute the QEA,

$$
\begin{aligned}
{\left[L^{i j}, L^{k l}\right] } & =\mathrm{i}\left(\delta_{j l} L^{i k}+\delta_{i k} L^{j l}-\delta_{j k} L^{i l}-\delta_{i l} L^{j k}\right), \\
{\left[L^{i j}, \phi^{k}\right] } & =-\mathrm{i}\left(\delta_{j k} \phi^{i}-\delta_{i k} \phi^{j}\right), \\
{\left[\phi^{i}, \phi^{j}\right] } & =\frac{1}{N} \mathrm{i} L^{i j} .
\end{aligned}
$$

This is the Lie algebra of $\mathrm{SO}(N+1)$. A curious fact of these relations is that, in the large $N$ limit, this Lie algebra is the same as that of the traditional theory, which can also be understood through the Wigner-Inönü contraction [69].

\section{Lattice Gauge Theories}

We now turn our attention to QEAs that arise in pure lattice gauge theories without matter fields. Consider a theory with a gauge group $G$, described by the Kogut-Susskind Hamiltonian [70],

$$
H=\frac{N}{\beta} \sum_{\left\langle\mathbf{r}, \mathbf{r}^{\prime}\right\rangle}\left(L_{\mathbf{r r}^{\prime}}^{a 2}+R_{\mathbf{r r}^{\prime}}^{a 2}\right)-\frac{\beta}{2 N} \sum_{\square}\left(U_{\square}+U_{\square}^{+}\right)
$$

where the summation is over links $\left\langle\mathbf{r}, \mathbf{r}^{\prime}\right\rangle$ and plaquettes $\square$. Unlike the spin models, now the quantum degrees of freedom live on links and consist of the electric fields $L^{a}, R^{a}$, $a=1,2, \cdots, \operatorname{dim} G$, and fundamental link operators $U_{i j}, i, j=1,2, \cdots, N$, where $\operatorname{dim} G$ is the dimension of the group $G$, and $N$ is the dimension of the fundamental representation of $G$. This choice of $U_{i j}$ is natural if we wish to couple our theory to matter fields in the fundamental representation at a later stage. As before, we have suppressed the the position indices since they do not play a role in the qubit regularization procedure or in the QEA that emerges.

In order to preserve the gauge symmetry, it is important that these gauge field operators satisfy the following extended symmetry algebra:

$$
\begin{aligned}
& {\left[L^{a}, L^{b}\right]=\mathrm{i} f^{a b c} L^{c}, \quad\left[L^{a}, U_{i j}\right]=T_{i k}^{a} U_{k j,}} \\
& {\left[R^{a}, R^{b}\right]=\mathrm{i} f^{a b c} R^{c}, \quad\left[R^{a}, U_{i j}\right]=-U_{i k} T_{k j}^{a}, \quad\left[L^{a}, R^{b}\right]=0 .}
\end{aligned}
$$

where $T^{a}$ are the generators of $G$ in the fundamental representation. In traditional lattice gauge theories, the Hilbert space again is the space of square integrable functions 
on the group manifold $G$, denoted as $L^{2}(G)$. The link operators $U_{i j}$ can be viewed as "position" operators on $G$, while $L^{a}$ and $R^{a}$ are "momentum" operators that generate left and right translation on $G$. Therefore, we further have the following relations among the $U_{i j}$ operators,

$$
\left[U_{i j}, U_{k l}\right]=0, \quad\left[U_{i j}, U_{k l}^{\dagger}\right]=0, \quad U^{\dagger} U=1,
$$

which are analogous to the relations Equation (4) in the $\mathrm{O}(N)$ models. Furthermore, the traditional Hilbert space naturally implies the relation

$$
\sum_{a}\left(L^{a}\right)^{2}=\sum_{a}\left(R^{a}\right)^{2},
$$

because, when we expand the Hilbert space in terms of irreps of the symmetry group (as discussed below in Equation (42)), both $\sum_{a}\left(L^{a}\right)^{2}$ and $\sum_{a}\left(R^{a}\right)^{2}$ are the Casimir operators labeling pairs of dual irreps. Thus, the extra relations in Equations (38) and (39) arise from the choice of the Hilbert space and are not related to the gauge symmetry of the model. In addition to these relations, the physical Hilbert space of the problem is much smaller and is obtained by imposing the Gauss' law. We will not worry about this issue here because we are only focusing on the local Hilbert space structure, and Gauss' law can be imposed after qubit regularizing the Hilbert space.

Qubit regularization of gauge theories consists of defining field operators $L_{Q^{\prime}}^{a} R_{Q}^{a}$ and $U_{i j}^{\mathrm{Q}}$ that act on a finite dimensional Hilbert space but preserve the gauge invariance by satisfying the extended symmetry algebra Equation (37). The additional relations Equations (38) and (39) of the traditional models can be sacrificed if necessary. To accomplish this, let us first understand the Hilbert space structure of the traditional lattice gauge theory. As in the spin models, we can choose a basis of "position" eigenstates $|g\rangle$, where $g \in G$ is an element of the group. They form a complete orthonormal basis of $L^{2}(G)$, i.e.,

$$
\left\langle g \mid g^{\prime}\right\rangle=\delta\left(g-g^{\prime}\right), \quad \int \mathrm{d} g|g\rangle\langle g|=1,
$$

where $\mathrm{d} g$ is the Haar measure on the group manifold. In this basis, all the link operators $U_{i j}$ are diagonal,

$$
U_{i j}|g\rangle=D_{i j}^{f}(g)|g\rangle,
$$

where $D^{f}(g)$ is an $N \times N$ matrix that corresponds to the fundamental representation of $g$.

In order to qubit-regularize the theory while preserving gauge invariance, we have to go from the "position" eigenstates to "momentum" eigenstates, where the infinite dimensional local Hilbert space is decomposed into a direct sum of irreps of the symmetry group. This can be accomplished using the Peter-Weyl theorem, which states that $L^{2}(G)$ decomposes into irreps of $G_{L} \times G_{R}$ as [71]

$$
L^{2}(G)=\bigoplus_{\lambda \in \hat{G}} V_{\lambda} \otimes V_{\lambda}^{*}
$$

where $\hat{G}$ denotes the set of irreps of $G$. Furthermore, the Peter-Weyl theorem also tells us that the space labeled the irrep $\lambda$ is spanned by the orthonormal basis $\left|D_{i j}^{\lambda}\right\rangle$,

$$
\left\langle D_{i j}^{\lambda} \mid D_{k l}^{\lambda^{\prime}}\right\rangle=\delta_{\lambda \lambda^{\prime}} \delta_{i k} \delta_{j l}, \quad \sum_{\lambda \in \hat{G}} \sum_{i, j}\left|D_{i j}^{\lambda}\right\rangle\left\langle D_{i j}^{\lambda}\right|=1,
$$

and are related to the "position" eigenstates by

$$
\left\langle g \mid D_{i j}^{\lambda}\right\rangle=\sqrt{d_{\lambda}} D_{i j}^{\lambda}(g),
$$


where $D^{\lambda}(g)$ is the matrix representation of $g$ in the irrep $\lambda$, and $d_{\lambda}$ is the dimension of the representation $\lambda$. For example, the state $|0\rangle$ in the trivial representation satisfies $\langle g \mid 0\rangle=1$. The operators $L^{a}$ and $R^{a}$ are block diagonal in this basis with matrix elements

$$
\left\langle D_{i j}^{\lambda}\left|L^{a}\right| D_{k l}^{\lambda}\right\rangle=\left(T_{\lambda}^{a}\right)_{i k} \delta_{j l}, \quad\left\langle D_{i j}^{\lambda}\left|R^{a}\right| D_{k l}^{\lambda}\right\rangle=-\left(T_{\lambda}^{a}\right)_{l j} \delta_{i k}
$$

where $T_{\lambda}^{a}$ are the corresponding generators of $G$ in the representation $\lambda$. On the other hand, when $U_{i j}$ acts on $\left|D_{i j}^{\lambda}\right\rangle$, states in other irreps are generated. This can be understood by noting that

$$
U_{k l}\left|D_{i j}^{\lambda}\right\rangle=\int \mathrm{d} g \sqrt{d_{\lambda}} D_{i j}^{\lambda}(g) D_{k l}^{f}(g)|g\rangle
$$

where $D_{i j}^{\lambda}(g) D_{k l}^{f}(g)$ is an element in the tensor product representation $\lambda \otimes f$. In the case of $G=\mathrm{SU}(N)$, we know that all irreps can be labeled by the Young diagrams. In particular, the fundamental representation $\mathbf{N}$ is labeled by a single box $\square$, and $\lambda \otimes f$ decomposes into irreps that correspond to adding a single box to the Young diagram of $\lambda$.

In order to qubit-regularize the theory, we will project the full Hilbert space given in Equation (42) to a finite dimensional one that only contains some of the irreps $\lambda$. While there are many choices, let us focus on a simple regularization scheme by choosing only the anti-symmetric representations,

$$
Q=\left\{\mathbf{N}, \bigwedge^{2} \mathbf{N}, \cdots, \bigwedge^{N} \mathbf{N} \cong \mathbf{1}\right\}
$$

where $\wedge^{k} \mathbf{N}$ is the irrep corresponding to Young diagrams with $k$ boxes arranged in a single column. All the other representations will be projected out. The action of $U$ on such a regularized Hilbert space has a simple form and is shown diagrammatically in Figure 1.

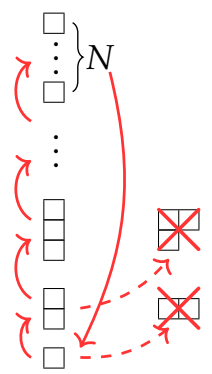

Figure 1. A simple qubit regularizarion scheme for the $\mathrm{SU}(N)$ gauge theory by keeping only the anti-symmetric representations. In this scheme, when $U$ acts on an irrep by adding a single box, only the irrep with a single column is kept, and the other is discarded (shown as a crossed-out irrep in the figure). Therefore, in this scheme, $U$ acts as a cyclic permutation on the anti-symmetric representations.

As we can see, $U$ acts cyclically in the space of irreps because the irrep with $N$ boxes in a column is the trivial irrep and adding a box takes to the trivial irrep takes you back to the irrep with a single box. This regularization scheme is particularly simple for $N=2,3$ because all matrix elements of $U$ in the regularized space can be written as certain integrals over $\mathrm{SU}(N)$ which can be evaluated simply using the invariance of the Haar measure, without the need to perform explicit integration over the group. For example, in the case of 
$\mathrm{SU}(2)$, there are only two irreps in this simple regularization scheme, the singlet and the fundamental $\square$. Then, there are two non-zero matrix elements given by

$$
\begin{aligned}
& \left\langle D_{i j}^{f}\left|U_{k l}\right| 0\right\rangle=\sqrt{2} \int \mathrm{d} g D_{i j}^{f}(g)^{*} D_{k l}^{f}(g)=\frac{1}{\sqrt{2}} \delta_{i k} \delta_{j l} . \\
& \left\langle 0\left|U_{k l}\right| D_{i j}^{f}\right\rangle=\sqrt{2} \int \mathrm{d} g D_{k l}^{f}(g) D_{i j}^{f}(g)=\frac{1}{\sqrt{2}} \varepsilon_{k i} \varepsilon_{l j} .
\end{aligned}
$$

In the case of SU(3), there are three irreps, the singlet, the fundamental $\square$ and antifundamental $\boxminus$. In this case, we need three matrix elements:

$$
\begin{aligned}
\left\langle D_{i j}^{f}\left|U_{k l}\right| 0\right\rangle & =\sqrt{3} \int \mathrm{d} g D_{i j}^{f}(g)^{*} D_{k l}^{f}(g)=\frac{1}{\sqrt{3}} \delta_{i k} \delta_{j l}, \\
\left\langle D_{i j}^{\bar{f}}\left|U_{k l}\right| D_{m n}^{f}\right\rangle & =3 \int \mathrm{d} g D_{i j}^{f}(g) D_{k l}^{f}(g) D_{m n}^{f}(g)=\frac{1}{2} \varepsilon_{i k m} \varepsilon_{j l n}, \\
\left\langle 0\left|U_{k l}\right| D_{i j}^{\bar{f}}\right\rangle & =\sqrt{3} \int \mathrm{d} g D_{k l}^{f}(g) D_{i j}^{f}(g)^{*}=\frac{1}{\sqrt{3}} \delta_{k i} \delta_{l j} .
\end{aligned}
$$

Further details on how to evaluate the above integrals are given in Appendix B. Armed with these results, we derive the QEAs for our simple regularization scheme in the case of $G=\mathrm{SU}(2)$ and $G=\mathrm{SU}(3)$.

\subsection{SU(2) Lattice Gauge Theory}

Using the fact $L^{2}(\mathrm{SU}(2))=L^{2}\left(S^{3}\right)$, we expect the QEA for $\mathrm{SU}(2)$ lattice gauge theory to be the same as that of the $\mathrm{O}(4)$ spin model when the two subspaces are regularized in the same way. In particular, when truncated to the trivial and fundamental representations, the QEA is expected to be $\mathrm{SO}(5)$. Let us derive this again here but from the perspective of a lattice gauge theory. The local quantum fields of the traditional model are given by $L^{a}, R^{a}, a=1,2,3$ and $U_{i j}, i, j=1,2$. Let $P_{Q}$ be a projector into a subspace of the irreps

$$
P_{Q}=\sum_{\lambda \in Q} \sum_{i, j}\left|D_{i j}^{\lambda}\right\rangle\left\langle D_{i j}^{\lambda}\right|,
$$

Using $P_{Q}$, we can define the qubit-regularized fields $L_{Q}^{a}, R_{Q}^{a}$ and $U_{i j}^{Q}$ as

$$
L_{Q}^{a}=P_{Q} L^{a} P_{Q}, \quad R_{Q}^{a}=P_{Q} R^{a} P_{Q}, \quad U_{i j}^{Q}=P_{Q} U_{i j} P_{Q} .
$$

For our simple choice of qubit regularization given in Equation (47), we get $Q=\{\mathbf{2}, \mathbf{1}\}$, i.e., the fundamental representation and the trivial representation. We can compute all the matrix elements of $L_{Q^{\prime}}^{a}, R_{Q}^{a}$ using Equation (45), and in the basis $\left(\left|D_{11}^{f}\right\rangle,\left|D_{12}^{f}\right\rangle,\left|D_{21}^{f}\right\rangle,\left|D_{22}^{f}\right\rangle,|0\rangle\right)^{T}$, we get the following $5 \times 5$ matrices:

$$
\begin{aligned}
L_{Q}^{1} & =\left(\begin{array}{lllll}
0 & 0 & 1 & 0 & 0 \\
0 & 0 & 0 & 1 & 0 \\
1 & 0 & 0 & 0 & 0 \\
0 & 1 & 0 & 0 & 0 \\
0 & 0 & 0 & 0 & 0
\end{array}\right), \quad L_{Q}^{2}=\left(\begin{array}{ccccc}
0 & 0 & -\mathrm{i} & 0 & 0 \\
0 & 0 & 0 & -\mathrm{i} & 0 \\
\mathrm{i} & 0 & 0 & 0 & 0 \\
0 & \mathrm{i} & 0 & 0 & 0 \\
0 & 0 & 0 & 0 & 0
\end{array}\right), L_{Q}^{3}=\left(\begin{array}{ccccc}
1 & 0 & 0 & 0 & 0 \\
0 & 1 & 0 & 0 & 0 \\
0 & 0 & -1 & 0 & 0 \\
0 & 0 & 0 & -1 & 0 \\
0 & 0 & 0 & 0 & 0
\end{array}\right), \\
R_{Q}^{1} & =\left(\begin{array}{ccccc}
0 & -1 & 0 & 0 & 0 \\
-1 & 0 & 0 & 0 & 0 \\
0 & 0 & 0 & -1 & 0 \\
0 & 0 & -1 & 0 & 0 \\
0 & 0 & 0 & 0 & 0
\end{array}\right), R_{Q}^{2}=\left(\begin{array}{ccccc}
0 & -\mathrm{i} & 0 & 0 & 0 \\
\mathrm{i} & 0 & 0 & 0 & 0 \\
0 & 0 & 0 & -\mathrm{i} & 0 \\
0 & 0 & \mathrm{i} & 0 & 0 \\
0 & 0 & 0 & 0 & 0
\end{array}\right), R_{Q}^{3}=\left(\begin{array}{ccccc}
-1 & 0 & 0 & 0 & 0 \\
0 & 1 & 0 & 0 & 0 \\
0 & 0 & -1 & 0 & 0 \\
0 & 0 & 0 & 1 & 0 \\
0 & 0 & 0 & 0 & 0
\end{array}\right) .
\end{aligned}
$$


Similarly, $U_{i j}^{Q}$ can be computed as $5 \times 5$ matrices using Equation (48),

$$
\begin{aligned}
& U_{11}^{Q}=\frac{1}{\sqrt{2}}\left(\begin{array}{ccccc}
0 & 0 & 0 & 0 & 1 \\
0 & 0 & 0 & 0 & 0 \\
0 & 0 & 0 & 0 & 0 \\
0 & 0 & 0 & 0 & 0 \\
0 & 0 & 0 & 1 & 0
\end{array}\right), \quad U_{12}^{Q}=\frac{1}{\sqrt{2}}\left(\begin{array}{ccccc}
0 & 0 & 0 & 0 & 0 \\
0 & 0 & 0 & 0 & 1 \\
0 & 0 & 0 & 0 & 0 \\
0 & 0 & 0 & 0 & 0 \\
0 & 0 & -1 & 0 & 0
\end{array}\right), \\
& U_{21}^{Q}=\frac{1}{\sqrt{2}}\left(\begin{array}{ccccc}
0 & 0 & 0 & 0 & 0 \\
0 & 0 & 0 & 0 & 0 \\
0 & 0 & 0 & 0 & 1 \\
0 & 0 & 0 & 0 & 0 \\
0 & -1 & 0 & 0 & 0
\end{array}\right), \quad U_{22}^{Q}=\frac{1}{\sqrt{2}}\left(\begin{array}{ccccc}
0 & 0 & 0 & 0 & 0 \\
0 & 0 & 0 & 0 & 0 \\
0 & 0 & 0 & 0 & 0 \\
0 & 0 & 0 & 0 & 1 \\
1 & 0 & 0 & 0 & 0
\end{array}\right) .
\end{aligned}
$$

Notice that $U_{11}^{Q \dagger}=U_{22}^{Q}$ and $U_{12}^{Q \dagger}=-U_{21}^{Q}$, which means that $U_{i j}^{Q^{+}}$are not an independent operators. These relations arise due to the fact that the fundamental representation of $\mathrm{SU}(2)$ is self-dual. It can be checked directly that $L_{Q}^{a}, R_{Q}^{a}$ and the independent Hermitian matrices constructed using $U_{i j}^{Q}$ form $\mathrm{SO}(5)$ algebra, as we already mentioned at the beginning of this section. As we will see below, this algebra is different from the more general pattern followed by $\mathrm{SU}(N)$ gauge theories with $N \geq 3$ because the fundamental representation of $\mathrm{SU}(N \geq 3)$ is not self-dual.

\subsection{SU(3) Lattice Gauge Theory}

We can extend the above calculations to $\mathrm{SU}(3)$ lattice gauge theory, which is interesting from the perspective of QCD and is already being explored on a quantum computer [62]. In this case, $P_{Q}$ is again of the form

$$
P_{Q}=\sum_{\lambda \in Q} \sum_{i, j}\left|D_{i j}^{\lambda}\right\rangle\left\langle D_{i j}^{\lambda}\right|,
$$

where, instead of Equation (47), we first choose the simpler case $Q=\{\mathbf{1}, \mathbf{3}\}$, i.e., the trivial representation and the fundamental representation. In this case, qubit-regularized Hilbert space is ten-dimensional with basis states

$$
\left(\left|D_{11}^{f}\right\rangle,\left|D_{12}^{f}\right\rangle,\left|D_{13}^{f}\right\rangle,\left|D_{21}^{f}\right\rangle,\left|D_{22}^{f}\right\rangle,\left|D_{23}^{f}\right\rangle,\left|D_{31}^{f}\right\rangle,\left|D_{32}^{f}\right\rangle,\left|D_{33}^{f}\right\rangle,|0\rangle\right)^{T} .
$$

Again using Equation (45), we can compute all matrix elements of $L_{Q^{\prime}}^{a} R_{Q}^{a}$ in this tendimensional space, which can be written compactly as

$$
L_{Q}^{a}=\lambda_{a} \otimes \mathbb{1}_{3} \oplus 0, \quad R_{Q}^{a}=-\mathbb{1}_{3} \otimes \lambda_{a}^{*} \oplus 0,
$$

where $\lambda_{a}, a=1, \cdots, 8$ are Gell-Mann matrices. Similarly, $U_{i j}^{Q}$ can also be constructed as $10 \times 10$ matrices using Equation (49). Using GAP, we can show that $L_{Q^{\prime}}^{a} R_{Q^{\prime}}^{a}, U_{i j}^{Q}$ and $U_{i j}^{Q+}$ generate the $\mathrm{SU}(10)$ algebra. In other words, starting with these 34 matrices, we generate 65 more matrices under commutation relations and obtain the 99 generators of SU(10). We will prove this more generally for the $\mathrm{SU}(N)$ lattice gauge theories in the next section.

We end this section by considering two more regularization schemes. If we choose $Q=\{\mathbf{1}, \mathbf{3}, \overline{\mathbf{3}}\}$ and input the matrices into GAP, we find the QEA to be $\mathrm{SU}(19)$. A nice feature of this regularization scheme is that $U_{i j}^{Q}$ is a cyclic raising operator in the representation space: $\mathbf{1} \rightarrow \mathbf{N} \rightarrow \overline{\mathbf{N}} \rightarrow \mathbf{1}$. This could be a desirable property since it is similar to that of the traditional model. Another simple scheme is obtained if we choose $Q=\{\mathbf{3}, \overline{\mathbf{3}}\}$, where GAP tells us that QEA is $\mathrm{Sp}(9)$. 


\section{3. $\mathrm{SU}(N)$ Lattice Gauge Theory}

In this section, we will generalize some of the results of $\mathrm{SU}(3)$ gauge theory to $\mathrm{SU}(N)$ gauge theories for $N \geq 3$. For example, we will show that, if we qubit-regularize an $\mathrm{SU}(N)$ gauge theory with $Q=\{\mathbf{1}, \mathbf{N}\}$, the resulting $Q E A$ is $S U\left(N^{2}+1\right)$. We will also provide a physical interpretation of all the elements in the Lie algebra of $\mathrm{SU}\left(N^{2}+1\right)$.

In this case, we can label the $N^{2}+1$ orthonormal basis states of the regularized Hilbert space as $|\alpha\rangle, \alpha=0,1,2, \cdots, N^{2}$, such that $|0\rangle$ denotes the state in the singlet, and $\left|D_{i j}^{f}\right\rangle=:|\alpha=N(i-1)+j\rangle$. We also define $U_{i j}^{Q}=: U_{\alpha}^{Q}$, where $1 \leq \alpha=N(i-1)+j \leq N^{2}$. The result in Appendix B implies that $\left\langle\gamma\left|U_{\alpha}^{Q}\right| \beta\right\rangle=\frac{1}{\sqrt{N}} \delta_{\gamma \alpha} \delta_{0 \beta}$, which gives

$$
U_{\alpha}^{Q}=\frac{1}{\sqrt{N}} e_{\alpha 0}, \quad U_{\alpha}^{Q^{+}}=\frac{1}{\sqrt{N}} e_{0 \alpha},
$$

where $e_{\alpha \beta}$ is an $\left(N^{2}+1\right) \times\left(N^{2}+1\right)$ matrix with matrix elements $\left(e_{\alpha \beta}\right)_{\gamma \delta}=\delta_{\alpha \gamma} \delta_{\beta \delta}$. Using this, we can show the following commutation relations:

$$
\left[U_{\alpha}^{\mathrm{Q}}, U_{\beta}^{\mathrm{Q}}\right]=\left[U_{\alpha}^{\mathrm{Q \dagger}}, U_{\beta}^{\mathrm{Q \dagger}}\right]=0, \quad\left[U_{\alpha}^{\mathrm{Q}}, U_{\beta}^{\mathrm{Q \dagger}^{\dagger}}\right]=\frac{1}{N}\left(e_{\alpha \beta}-\delta_{\alpha \beta} e_{00}\right) .
$$

Interestingly, we again observe that this algebra is reduced to the traditional one in Equation (38) when $N \rightarrow \infty$. $\left[U_{\alpha}^{Q}, U_{\beta}^{Q^{+}}\right]$generate $N^{4}$ operators, each of which is an $\left(N^{2}+1\right) \times\left(N^{2}+1\right)$ matrix. The linear combinations of these operators give all traceless matrices within the $N^{2} \times N^{2}$ block that does not contain the singlet. More concretely, $\left[U_{\alpha}^{Q}, U_{\beta}^{Q \dagger}\right] \propto e_{\alpha \beta}$ for $\alpha \neq \beta$ give all off diagonal elements in this $N^{2} \times N^{2}$ block, while $\left[U_{\alpha}^{\mathrm{Q}}, U_{\alpha}^{\mathrm{O}^{\dagger}}\right]-\left[U_{\alpha+1}^{\mathrm{Q}}, U_{\alpha+1}^{\mathrm{Q \dagger}}\right]=e_{\alpha \alpha}-e_{(\alpha+1)(\alpha+1)}$ for $\alpha=1, \cdots, N^{2}-1$ gives the $N^{2}-1$ independent traceless diagonal matrices. We denote these $N^{4}-1$ operators which are represented by traceless matrices within the $N^{2} \times N^{2}$ block as $t_{\alpha \beta}$ such that

$$
t_{\alpha \beta}= \begin{cases}e_{\alpha \beta} & \text { when } \alpha \neq \beta, \\ e_{\alpha \alpha}-e_{(\alpha+1)(\alpha+1)} & \text { when } \alpha=\beta \neq N^{2}\end{cases}
$$

The Hermitian linear combinations of these matrices form the Lie algebra of $\mathrm{SU}\left(N^{2}\right)$. There is one more independent operator

$$
E^{Q}:=\frac{N}{N^{2}+1} \sum_{\alpha=1}^{N^{2}}\left[U_{\alpha}^{Q}, U_{\alpha}^{Q \dagger}\right]=\frac{1}{N^{2}+1}-e_{00},
$$

which is normalized such that $U_{\alpha}^{Q}$ and $U_{\alpha}^{Q+}$ have charges \pm 1 in Equation (62). $E^{Q}$ is traceless and proportional to the identity in the $N^{2} \times N^{2}$ block. The $2 N^{2}$ operators $U_{\alpha}^{Q}$ and $U_{i}^{Q+}$, along with $N^{4}-1$ operators $t_{\alpha \beta}$ and the operator $E^{Q}$, form the $N^{4}+2 N^{2}$ dimensional Lie algebra of $\mathrm{SU}\left(N^{2}+1\right)$.

The operator $E^{Q}$ is the unique element in the Lie algebra of $\mathrm{SU}\left(N^{2}+1\right)$ that commutes with all $L^{a}$ and $R^{a}$, which can be argued as follows. The Hilbert space is a direct sum of the $N^{2}$ basis states $\left|D_{i j}^{f}\right\rangle$ and the singlet $|0\rangle$, which are two irreps of $\mathrm{SU}(N)_{L} \times \mathrm{SU}(N)_{R}$. Using Schur's lemma, we know that any element that commutes with both $L^{a}$ and $R^{a}$ must be proportional to identity in each of the irreps. Since the generators of $\mathrm{SU}\left(N^{2}+1\right)$ are all traceless, there is a unique element that commutes with both $L^{a}$ and $R^{a}$, which is nothing but the $E^{Q}$ operator. Furthermore, it is easy to verify that $E^{Q}$ also satisfies

$$
\left[E^{Q}, U_{i j}^{Q}\right]=U_{i j}^{Q}, \quad\left[E^{Q}, U_{i j}^{Q^{\dagger}}\right]=-U_{i j}^{Q^{+}} .
$$


Therefore, the operator $E^{Q}$ can be viewed as the generator of a $\mathrm{U}(1)$ gauge field, and the link operators $U_{i j}^{Q}$ and $U_{i j}^{Q \dagger}$ carry opposite charges under this $\mathrm{U}(1)$ gauge field. Thus, $Q=\{\mathbf{1}, \mathbf{N}\}$ could also be used as a qubit regularization of a $\mathrm{U}(N)$ gauge theory. This is reminiscent of the D-theory approach where $\mathrm{SU}(N)$ gauge threory was implemented using the QEA of $S U(2 N)$ and again, in that regularization, there was an element similar to $E^{\mathrm{Q}}$ which could be used to construct a $\mathrm{U}(N)$ gauge theory also. However, the emergence of the $E^{Q}$ operator is not a general property. For example, there was no such operator in the case of the $\mathrm{SO}(5)$ scheme in $\mathrm{SU}(2)$ lattice gauge theory. We will also see that in the $\mathrm{SU}\left(N^{2}\right)$ scheme for $\mathrm{SU}(N)$ lattice gauge theory to be discussed below, the operator $E^{Q}$ does not emerge.

Let us now understand how the $N^{4}-1$ operators $t_{\alpha \beta}$ that appear in the Lie algebra of $\mathrm{SU}\left(N^{2}\right)$, transform under the $\mathrm{SU}(N)_{L} \times \mathrm{SU}(N)_{R}$ subgroup that is embedded in it. In the operators $t_{\alpha \beta}$, the index $\alpha$ transforms as $\mathbf{N}_{L} \otimes \overline{\mathbf{N}}_{R}$, while the index $\beta$ transforms as $\overline{\mathbf{N}}_{L} \otimes \mathbf{N}_{R}$. Hence, together with $E$, the operators $t_{\alpha \beta}$ transform as

$$
\begin{aligned}
\mathbf{N}_{L} \otimes \overline{\mathbf{N}}_{R} \otimes \overline{\mathbf{N}}_{L} \otimes \mathbf{N}_{R} & \cong(\mathbf{N} \otimes \overline{\mathbf{N}})_{L} \otimes(\overline{\mathbf{N}} \otimes \mathbf{N})_{R} \\
& \cong(\operatorname{adj} \oplus \mathbf{1})_{L} \otimes(\operatorname{adj} \oplus \mathbf{1})_{R} \\
& \cong \operatorname{adj}_{L} \otimes \operatorname{adj}_{R} \oplus \operatorname{adj}_{L} \oplus \operatorname{adj}_{R} \oplus \mathbf{1} .
\end{aligned}
$$

In this decomposition, $\operatorname{adj}_{L}$ and $\operatorname{adj}_{R}$ are nothing but $L^{a}$ and $R^{a}$, while $\mathbf{1}$ is the $E^{Q}$ operator. The remaining part $\operatorname{adj}_{L} \otimes \operatorname{adj}_{R}$ is exactly the way adjoint link operators $U_{i j}^{\text {adj, } Q}$ would transform. Thus, we also get the adjoint links for free.

In summary, we learn that the qubit regularization with the QEA SU $\left(N^{2}+1\right)$ naturally contains the electric operators $L_{Q}^{a}, R_{Q}^{a}$ and $E^{Q}$, the fundamental link operators $U_{i j}^{Q}$ and $\left(U_{i j}^{Q}\right)^{\dagger}$, and the adjoint link operators $U_{i j}^{\text {adj, } Q}$ that are self-dual. This discussion also motivates to define a qubit regularization of an $\mathrm{SU}(N)$ gauge theory with adjoint link operators using an $N^{2}$ dimensional Hilbert space, with the QEA of $\mathrm{SU}\left(N^{2}\right)$.

\section{Discussion and Conclusions}

Using examples from spin models and gauge theories, in this work, we showed that qubit regularizations are characterized by an algebraic structure referred to as the qubit emdedding algebra (QEA). We propose to use QEA along to define the qubit regularization scheme. For example, we showed how the traditional $\mathrm{O}(N)$ spin model can be qubit-regularized using the $\mathrm{SO}(N+1)$ scheme. We also showed that the traditional $\mathrm{SU}(2)$ gauge theory can be qubit-regularized using a $\mathrm{SO}(5)$ scheme, while the $\mathrm{SU}(N)$ gauge theory can be qubit-regularized using a $\mathrm{SU}\left(N^{2}+1\right)$ scheme for $N \geq 3$. For $N=3$, we discovered that there are also $\mathrm{Sp}(9)$ or $\mathrm{SU}(19)$ schemes, using the mathematical software package GAP. Based on these numerical results using GAP, we conjecture that, for $Q=\left\{\mathbf{1}, \mathbf{N}, \wedge^{2} \mathbf{N}, \cdots, \wedge^{N-1} \mathbf{N}\right\}$ the QEA is SU $\left(\left(\begin{array}{c}2 N \\ N\end{array}\right)-1\right)$, while for $Q=\{\mathbf{N}, \overline{\mathbf{N}}, \mathbf{1}\}$ and $N \geq 4$, the QEA is $\mathrm{SO}\left(2 N^{2}+1\right)$. The latter conjecture is based on the result from the $\mathrm{SU}(3)$ gauge theory that, if we choose $Q=\{\mathbf{3}, \overline{\mathbf{3}}, \mathbf{1}\}$ and set the matrix elements $\left\langle D_{i j}^{\bar{f}}\left|U_{k l}\right| D_{m n}^{f}\right\rangle$ to be zero, which vanishes for $N \geq 4$, then the QEA is $\mathrm{SO}(19)$.

The idea of qubit regularization was introduced long ago within the D-theory approach [43-45], but not within a systematic approach that we have adopted in our work here. Interestingly, some of the QEAs that were proposed earlier are the same as the ones we found here. For example, the $\mathrm{O}(N)$ spin models were also qubit-regularized using the QEA of $\mathrm{SO}(N+1)$ in [45]. Even the qubit regularization of the $\mathrm{SU}(2)$ gauge theory using the QEA of $\mathrm{SO}(5)$ was known earlier [43]. The representation proposed in the D-theory approach was a four-dimensional spin representation of $\mathrm{SO}(5)$, i.e., $\mathrm{Sp}(2)$, while the one we naturally found here from the traditional model is the five-dimensional fundamental representation. This is yet another important feature of QEA to keep in mind - the QEA alone does not fully determine the qubit regularization, we also need to specify the representation of the QEA that is used to construct the quantum fields. 
In the D-theory approach, $\mathrm{SU}(N)$ lattice gauge theories for $N \geq 3$ were qubitregularized using the QEA of $\mathrm{SU}(2 N)$ in the fundamental representation. On the other hand, in our work, we found regularization schemes with QEA of SU $\left(N^{2}+1\right)$ in the fundamental representation. Interestingly, in both of these regularization schemes, there is an additional operator $E^{Q}$ that generates $U(1)$ gauge transformations, which means that the same QEAs can also be used to regularize a $U(N)$ lattice gauge theory. Since the QEA is a closed algebra which always contains the sub-algebra of $\mathrm{SU}(N)_{L} \times \mathrm{SU}(N)_{R}$, all operators in the QEA form irreps of $\mathrm{SU}(N)_{L} \times \mathrm{SU}(N)_{R}$. The presence of $E^{Q}$ in the QEA depends on whether there is an operator in the QEA that transforms as a singlet under $\mathrm{SU}(N)_{L} \times \mathrm{SU}(N)_{R}$. We also showed how, in our $\mathrm{SU}\left(N^{2}+1\right)$ regularization scheme, the adjoint link operators are also generated naturally, and in a gauge theory with only adjoint link operators, we can simply regularize the theory with the $\mathrm{SU}\left(N^{2}\right)$ scheme. In this case, no new operators are generated.

A significant distinction between the regularization schemes discussed in this paper as compared to earlier schemes [43-45] is that the relation $\sum_{a}\left(L^{a}\right)^{2}=\sum_{a}\left(R^{a}\right)^{2}$ in Equation (39) is preserved in the former but not in the latter. In traditional lattice gauge theories, this relation arises from the fact that both $\sum_{a}\left(L^{a}\right)^{2}$ and $\sum_{a}\left(R^{a}\right)^{2}$ are Casimir operators which label the representations in Equation (42) and they are equal. Our regularization scheme preserves this structure of the irreps naturally, but not the quantum link model. Further studies are necessary to understand whether this difference is important to recover the asymptotically free fixed point.

Author Contributions: Conceptualization, S.C.; methodology, S.C. and H.L.; software, H.L.; validation, S.C. and H.L.; formal analysis H.L.; writing — original draft, H.L.; writing—review and editing, S.C. and H.L. All authors have read and agreed to the published version of the manuscript.

Funding: The material presented here is based on work supported by the U.S. Department of Energy, Office of Science-High Energy Physics Contract KA2401032 (Triad National Security, LLC Contract Grant No. 89233218CNA000001) to Los Alamos National Laboratory. S.C. is supported by a Duke subcontract of this grant. S.C. and H.L. are also supported for this work in part by the U.S. Department of Energy, Office of Science, Nuclear Physics program under Award No. DE-FG02-05ER41368.

Institutional Review Board Statement: Not applicable.

Informed Consent Statement: Not applicable.

Data Availability Statement: Not applicable.

Acknowledgments: We would like to thank Tanmoy Bhattacharya, Rajan Gupta, Austin Hulse, Iman Marvian, Mendel Nguyen, Hersh Singh, and Uwe-Jens Wiese for discussions. We also thank Wiese for sharing his notes with us after our manuscript was completed. From these notes we learned that some of our results were also independently discovered by him. We would also like to thank MathOverflow user Paul Levy (https: / / mathoverflow.net/users/26635/paul-levy, accessed on 21 November 2021), for sketching out the idea for the proof of Theorem A1. The material presented here is based on work supported by the U.S. Department of Energy, Office of Science-High Energy Physics Contract KA2401032 (Triad National Security, LLC Contract Grant No. 89233218CNA000001) to Los Alamos National Laboratory. S.C. is supported by a Duke subcontract of this grant. S.C. and H.L. are also supported for this work in part by the U.S. Department of Energy, Office of Science, Nuclear Physics program under Award No. DE-FG02-05ER41368.

Conflicts of Interest: The authors declare no conflict of interest.

\section{Appendix A. Proof of the QEAs for the $\mathrm{O}(2)$ Model}

Let us formulate the result as the following theorem:

Theorem A1. Let $\phi_{d}^{+}$and $\phi_{d}^{-}$be the $d \times d$ upper shift matrix and lower shift matrix, respectively, i.e., $\left(\phi_{d}^{ \pm}\right)_{i j}=\delta_{i \pm 1, j}$, where $1 \leq i, j \leq d$. Let $\mathfrak{g}_{d}$ be the Lie algebra generated by $\phi_{d}^{+}$and $\phi_{d}^{-}$over 
C. Let $J_{d}=(-1)^{i} \delta_{i, d+1-i}$ be an anti-diagonal bilinear form. Then, $\mathfrak{g}_{d}$ is the Lie algebra of $d \times d$ matrices that preserve the bilinear form $J_{d}$, i.e.,

$$
\mathfrak{g}=\left\{x \in \mathfrak{g l}(d, \mathbb{C}) \mid x^{T} J_{d}+J_{d} x=0\right\} .
$$

In particular, since $J_{d}$ is symmetric (anti-symmetric) when $d$ is odd (even), we know $\mathfrak{g}_{d} \cong \mathfrak{s o}(d, \mathbb{C})$ $\left(\mathfrak{g}_{d} \cong \mathfrak{s p}(d, \mathbb{C})\right)$ when $d$ is odd (even).

Proof. Let us assume that $d$ is odd, and the case of $d$ being even can be proved in a similar way. It is easy to check that $\phi_{d}^{ \pm T} J_{d}+J_{d} \phi_{d}^{ \pm}=0$. Therefore, $\mathfrak{g}_{d} \subset \mathfrak{s o}(d, \mathbb{C})$. In order to show $\mathfrak{g}_{d} \cong \mathfrak{s o}(d, \mathbb{C})$, we use induction over $d$. When $d=3$, we have

$$
h_{3}:=\left[\phi_{3}^{+}, \phi_{3}^{-}\right]=\left(\begin{array}{ccc}
1 & 0 & 0 \\
0 & 0 & 0 \\
0 & 0 & -1
\end{array}\right) \text {. }
$$

Clearly, $\phi_{3}^{+}, \phi_{3}^{-}, h_{3}$ are independent of each other, and therefore $\operatorname{dim}_{\mathbb{C}}\left(\mathfrak{g}_{3}\right) \geq 3$. Since $\operatorname{dim}_{\mathbb{C}}(\mathfrak{s o}(3, \mathbb{C}))=3$, we know that $\mathfrak{g}_{3} \cong \mathfrak{s o}(3, \mathbb{C})$.

Now, assuming $\mathfrak{g}_{d-2} \cong \mathfrak{s o}(d-2, \mathbb{C})$, let us consider the case for $d$. In this case, we have

$$
h_{d}:=\left[\phi_{d}^{+}, \phi_{d}^{-}\right]=\left(\begin{array}{ccc}
1 & \mathbf{0} & 0 \\
\mathbf{0} & \mathbf{0}_{d-2} & \mathbf{0} \\
0 & \mathbf{0} & -1
\end{array}\right)
$$

and

$$
\begin{aligned}
\phi_{d}^{+}+\left[\phi_{d}^{+}, h_{d}\right] & =\left(\begin{array}{ccc}
0 & \mathbf{0} & 0 \\
\mathbf{0} & \phi_{d-2}^{+} & \mathbf{0} \\
0 & \mathbf{0} & 0
\end{array}\right), \\
\phi_{d}^{-}-\left[\phi_{d}^{-}, h_{d}\right] & =\left(\begin{array}{ccc}
0 & \mathbf{0} & 0 \\
\mathbf{0} & \phi_{d-2}^{-} & \mathbf{0} \\
0 & \mathbf{0} & 0
\end{array}\right) .
\end{aligned}
$$

Therefore, we see that $\mathfrak{g}_{d} \supset \mathfrak{s o}(d-2, \mathbb{C})$ using the induction hypothesis. We will complete the proof using the root system of $\mathfrak{s o}(d, \mathbb{C})$. In order to do so, we need to first determine the Cartan subalgebra of $\mathfrak{s o}(d, \mathbb{C})$, which can be chosen to be

$$
h_{d}^{i}:=e_{i i}-e_{d+1-i, d+1-i},
$$

where $e_{i j}$ is an $d \times d$ matrix with 1 at the $i j$ position and zero everywhere else. From this definition, we can see that $h_{d}^{1}=h_{d}$. Then, for an element $x \in \mathfrak{g}_{d}$ which simultaneously diagonalizes $h_{d}^{i}$ in the adjoint representation, i.e., $\left[h_{d}^{i}, x\right]=\alpha^{i} x$, the corresponding root is defined to be $\vec{\alpha}$. It can be checked that $\left[h_{d}, \phi_{d}^{+}\right]$simultaneously diagonalizes $h_{d}^{i}$ with eigenvalues $\vec{\alpha}=(1,-1,0, \cdots, 0)^{T}$. Together with the simple roots in $\mathfrak{s o}(d-2, \mathbb{C})$, i.e.,

$$
\left(\begin{array}{c}
0 \\
1 \\
-1 \\
0 \\
\vdots \\
0
\end{array}\right),\left(\begin{array}{c}
0 \\
0 \\
1 \\
-1 \\
\vdots \\
0
\end{array}\right), \cdots,\left(\begin{array}{c}
0 \\
\vdots \\
0 \\
0 \\
1 \\
-1
\end{array}\right),\left(\begin{array}{l}
0 \\
\vdots \\
0 \\
0 \\
0 \\
1
\end{array}\right),
$$

they form all the simple roots of $\mathfrak{s o}(d, \mathbb{C})$. Therefore, $\mathfrak{g}_{d} \cong \mathfrak{s o}(d, \mathbb{C})$. 


\section{Appendix B. Some Integrals over $\mathrm{SU}(N)$}

In this appendix, we evaluate some integrals over $\mathrm{SU}(N)$ using the invariance of the Haar measure rather than actually doing any integration explicitly, which will be useful to determine the matrix elements of $U_{i j}$ in the main text. Let us again formulate the results in the following theorem:

Theorem A2. Let $D^{f}(g)$ be an $N \times N$ matrix corresponding the fundamental representation of $g \in \mathrm{SU}(N)$. Let $\mathrm{d} g$ be the Haar measure over the group $\mathrm{SU}(N)$. Then, we have the following identities:

$$
\begin{aligned}
\int \mathrm{d} g D_{i j}^{f}(g)^{*} D_{k l}^{f}(g) & =\frac{1}{N} \delta_{i k} \delta_{j l}, \\
\int \mathrm{d} g D_{i_{i} j_{1}}^{f}(g) D_{i_{2} j_{2}}^{f}(g) \cdots D_{i_{N} j_{N}}^{f}(g) & =\frac{1}{N !} \varepsilon_{i_{1} i_{2} \cdots i_{N}} \varepsilon_{j_{1} j_{2} \cdots j_{N}} .
\end{aligned}
$$

Proof. The key observation in the proof is that the permutation matrices are in the group $\mathrm{O}(N)$. In particular, all even permutations are in the group $\mathrm{SO}(N)$ and hence $\mathrm{SU}(N)$, while, for odd permutations, upon changing one element from 1 to -1 , they are also in the group $\mathrm{SU}(N)$. Therefore, for $\sigma$ and $\tau$ being even permutations, we have $D^{f}\left(\sigma g \tau^{-1}\right)=$ $D^{f}(\sigma) D^{f}(g) D^{f}\left(\tau^{-1}\right)$, and thus $D_{i j}^{f}\left(\sigma g \tau^{-1}\right)=D_{\sigma(i) \tau(j)}^{f}(g)$.

First, let us focus on the integral Equation (A7). We know that $\operatorname{tr} D^{f}(g)^{\dagger} D^{f}(g)=N$ is a constant function on $\mathrm{SU}(N)$. Therefore,

$$
N=\int \mathrm{d} g \operatorname{tr} D^{f}(g)^{\dagger} D^{f}(g)=\sum_{i j} \int \mathrm{d} g D_{j i}^{f}(g)^{\dagger} D_{i j}^{f}(g)=\sum_{i j} \int \mathrm{d} g D_{i j}^{f}(g)^{*} D_{i j}^{f}(g) .
$$

Now, for some fixed $i, j$, let us choose two even permutations $\sigma$ and $\tau$ that satisfy $\sigma(i)=1$ and $\tau(j)=1$. Then, by changing the integration variable $g \mapsto \sigma g \tau^{-1}$, we have

$$
\begin{aligned}
\int \mathrm{d} g D_{i j}^{f}(g)^{*} D_{i j}^{f}(g) & =\int \mathrm{d}\left(\sigma g \tau^{-1}\right) D_{i j}^{f}\left(\sigma g \tau^{-1}\right)^{*} D_{i j}^{f}\left(\sigma g \tau^{-1}\right) \\
& =\int \mathrm{d}\left(\sigma g \tau^{-1}\right) D_{\sigma(i) \tau(j)}^{f}(g)^{*} D_{\sigma(i) \tau(j)}^{f}(g) \\
& =\int \mathrm{d} g D_{11}^{f}(g)^{*} D_{11}^{f}(g),
\end{aligned}
$$

where in the last line we replaced $\mathrm{d} \sigma g \tau^{-1}$ by $\mathrm{d} g$ using the invariance of the Haar measure. Therefore, we have

$$
\int \mathrm{d} g D_{i j}^{f}(g)^{*} D_{i j}^{f}(g)=\frac{1}{N^{2}} \sum_{i j} \int \mathrm{d} g D_{i j}^{f}(g)^{*} D_{i j}^{f}(g)=\frac{1}{N} .
$$

Since different matrix coefficients are orthogonal with respect to the integration over $\mathrm{SU}(N)$, we arrive at the result Equation (A7).

Now, let us prove Equation (A8). Similarly, we have $\operatorname{det} D^{f}(g)=1$ as a constant function on $\mathrm{SU}(N)$. Therefore,

$$
1=\int \mathrm{d} g \operatorname{det} D^{f}(g)=\sum_{\sigma \in S_{N}} \int \mathrm{d} g \operatorname{sgn}(\sigma) D_{1 \sigma(1)}^{f}(g) \cdots D_{N \sigma(N)}^{f}(g) .
$$

Again, we use the fact that the group $\mathrm{SU}(N)$ includes all even permutations. Let $\tau$ be an even permutation, and change the integration variable $g \mapsto g \tau^{-1}$, we have 


$$
\begin{aligned}
\int \mathrm{d} g \operatorname{sgn}(\sigma) D_{1 \sigma(1)}^{f}(g) \cdots D_{N \sigma(N)}^{f}(g) & =\int \mathrm{d}\left(g \tau^{-1}\right) \operatorname{sgn}(\sigma) D_{1 \sigma(1)}^{f}\left(g \tau^{-1}\right) \cdots D_{N \sigma(N)}^{f}\left(g \tau^{-1}\right) \\
& =\int \mathrm{d} g \operatorname{sgn}(\sigma) D_{1 \tau \circ \sigma(1)}^{f}(g) \cdots D_{N \tau \circ \sigma(N)}^{f}(g)
\end{aligned}
$$

where again we have used the invariance of the Haar measure. Then, if $\sigma$ is an even permutation, we can choose $\tau \circ \sigma=(1)$, while, if $\sigma$ is an odd permutation, we can choose $\tau \circ \sigma=(12)$, where we have used the cycle notation for permutations. Therefore,

$$
\int \mathrm{d} g\left(D_{11}^{f}(g) D_{22}^{f}(g)-D_{12}^{f}(g) D_{21}^{f}(g)\right) D_{33}^{f}(g) \cdots D_{N N}^{f}(g)=\frac{2}{N !} .
$$

Now, let us consider an element $h \in \mathrm{SU}(N)$ of the form $h=\mathrm{i} \sigma^{2} \oplus \mathbb{1}_{N-2}$. This element has the property that $D_{i 2}^{f}\left(g h^{-1}\right)=D_{i 1}^{f}(g)$ and $D_{i 1}^{f}\left(g h^{-1}\right)=-D_{i 2}^{f}(g)$, while all the other matrix elements $D_{i j}^{f}(g)$ are unchanged. Using this relation, we can show that

$$
\int \mathrm{d} g D_{11}^{f}(g) D_{22}^{f}(g) \cdots D_{N N}^{f}(g)=-\int \mathrm{d} g D_{12}^{f}(g) D_{21}^{f}(g) \cdots D_{N N}^{f}(g)=\frac{1}{N !},
$$

which implies

$$
\int \mathrm{d} g D_{1 \sigma(1)}^{f}(g) \cdots D_{N \sigma(N)}^{f}(g)=\frac{1}{N !} \operatorname{sgn}(\sigma) .
$$

Finally, from the weight vectors of the fundamental representation and its dual representation of $\mathrm{SU}(N)$, we see that the decomposition of $D_{i_{i} j_{1}}^{f}(g) D_{i_{2} j_{2}}^{f}(g) \cdots D_{i_{N} j_{N}}^{f}(g)$ into irreducible components contains a trivial representation only if all the $i^{\prime}$ s are distinct and all the $j$ 's are distinct. Therefore, we can write the result compactly as Equation (A8).

These formulas can be checked explicitly in the case of $\mathrm{SU}(2)$ and $\mathrm{SU}(3)$. A parameterization of $\mathrm{SU}(2)$ is given

$$
D^{f}=\left(\begin{array}{cc}
\cos \theta \mathrm{e}^{\mathrm{i} \phi} & \sin \theta \mathrm{e}^{\mathrm{i} \psi} \\
-\sin \theta \mathrm{e}^{-\mathrm{i} \psi} & \cos \theta \mathrm{e}^{-\mathrm{i} \phi}
\end{array}\right),
$$

where $\theta \in\left[0, \frac{\pi}{2}\right], \phi \in[0,2 \pi)$ and $\psi \in[0,2 \pi)$, and the Haar measure on it is $\frac{1}{2 \pi^{2}} \sin 2 \theta \mathrm{d} \theta \mathrm{d} \phi \mathrm{d} \psi$. The parameterization and Haar measure of $\mathrm{SU}(3)$ can be found in [72].

Now, let $\left|D^{f}\right\rangle$ be the normalized vector corresponding to $D^{f}$; then, schematically, we have the following matrix elements of $U$ when they are non-zero,

$$
\begin{aligned}
\left\langle D^{f}|U| 0\right\rangle & =\frac{1}{\sqrt{N}}, \\
\left\langle D^{f *}\left|U^{N-2}\right| D^{f}\right\rangle & = \pm \frac{1}{(N-1) !},
\end{aligned}
$$

which are what we used in the main text.

\section{References}

1. Ladd, T.D.; Jelezko, F.; Laflamme, R.; Nakamura, Y.; Monroe, C.; O’Brien, J.L. Quantum computers. Nature 2010, 464, 45-53. [CrossRef] [PubMed]

2. Troyer, M.; Wiese, U.J. Computational complexity and fundamental limitations to fermionic quantum Monte Carlo simulations. Phys. Rev. Lett. 2005, 94, 170201. [CrossRef] [PubMed]

3. Preskill, J. Simulating quantum field theory with a quantum computer. Proc. Sci. 2018, 334, 24. [CrossRef]

4. Jordan, S.P.; Lee, K.S.M.; Preskill, J. Quantum Algorithms for Quantum Field Theories. Science 2012, 336, 1130-1133. [CrossRef]

5. Jordan, S.P.; Lee, K.S.M.; Preskill, J. Quantum Computation of Scattering in Scalar Quantum Field Theories. Quant. Inf. Comput. 2014, 14, 1014-1080. [CrossRef] 
6. Jordan, S.P.; Lee, K.S.M.; Preskill, J. Quantum Algorithms for Fermionic Quantum Field Theories. arXiv 2014, arXiv:1404.7115.

7. Jordan, S.P.; Krovi, H.; Lee, K.S.M.; Preskill, J. BQP-completeness of Scattering in Scalar Quantum Field Theory. Quantum 2018, 2, 44. [CrossRef]

8. Dumitrescu, E.F.; McCaskey, A.J.; Hagen, G.; Jansen, G.R.; Morris, T.D.; Papenbrock, T.; Pooser, R.C.; Dean, D.J.; Lougovski, P. Cloud Quantum Computing of an Atomic Nucleus. Phys. Rev. Lett. 2018, 120, 210501. [CrossRef]

9. Lu, H.H.; Klco, N.; Lukens, J.M.; Morris, T.D.; Bansal, A.; Ekström, A.; Hagen, G.; Papenbrock, T.; Weiner, A.M.; Savage, M.J.; et al. Simulations of Subatomic Many-Body Physics on a Quantum Frequency Processor. Phys. Rev. A 2019, 100, 012320. [CrossRef]

10. Kokail, C.; Maier, C.; van Bijnen, R.; Brydges, T.; Joshi, M.K.; Jurcevic, P.; Muschik, C.A.; Silvi, P.; Blatt, R.; Roos, C.F.; et al. Self-verifying variational quantum simulation of lattice models. Nature 2019, 569, 355-360. [CrossRef]

11. Klco, N.; Dumitrescu, E.F.; McCaskey, A.J.; Morris, T.D.; Pooser, R.C.; Sanz, M.; Solano, E.; Lougovski, P.; Savage, M.J. Quantumclassical computation of Schwinger model dynamics using quantum computers. Phys. Rev. A 2018, 98, 032331. [CrossRef]

12. Klco, N.; Savage, M.J. Digitization of scalar fields for quantum computing. Phys. Rev. A 2019, 99, 052335. [CrossRef]

13. Lamm, H.; Lawrence, S. Simulation of Nonequilibrium Dynamics on a Quantum Computer. Phys. Rev. Lett. 2018, 121, 170501. [CrossRef]

14. Banerjee, D.; Dalmonte, M.; Muller, M.; Rico, E.; Stebler, P.; Wiese, U.J.; Zoller, P. Atomic Quantum Simulation of Dynamical Gauge Fields coupled to Fermionic Matter: From String Breaking to Evolution after a Quench. Phys. Rev. Lett. $2012,109,175302$. [CrossRef]

15. Bazavov, A.; Meurice, Y.; Tsai, S.W.; Unmuth-Yockey, J.; Zhang, J. Gauge-invariant implementation of the Abelian Higgs model on optical lattices. Phys. Rev. D 2015, 92, 076003. [CrossRef]

16. Unmuth-Yockey, J.F. Gauge-invariant rotor Hamiltonian from dual variables of 3D U(1) gauge theory. Phys. Rev. D 2019, 99, 074502. [CrossRef]

17. Meurice, Y. Examples of symmetry-preserving truncations in tensor field theory. Phys. Rev. D 2019, 100, 014506. [CrossRef]

18. Mathur, M. Harmonic oscillator prepotentials in SU(2) lattice gauge theory. J. Phys. A 2005, 38, 10015-10026. [CrossRef]

19. Anishetty, R.; Mathur, M.; Raychowdhury, I. Prepotential formulation of SU(3) lattice gauge theory. J. Phys. A 2010, $43,035403$. [CrossRef]

20. Raychowdhury, I.; Stryker, J.R. Solving Gauss's Law on Digital Quantum Computers with Loop-String-Hadron Digitization. Phys. Rev. Res. 2020, 2, 033039. [CrossRef]

21. Raychowdhury, I.; Stryker, J.R. Loop, string, and hadron dynamics in SU(2) Hamiltonian lattice gauge theories. Phys. Rev. D 2020, 101, 114502. [CrossRef]

22. Davoudi, Z.; Raychowdhury, I.; Shaw, A. Search for efficient formulations for Hamiltonian simulation of non-Abelian lattice gauge theories. Phys. Rev. D 2021, 104, 074505. [CrossRef]

23. Raychowdhury, I. Towards Quantum Simulating Non-Abelian Gauge Theories. arXiv 2021, arXiv:2106.11475. [CrossRef]

24. Banuls, M.C.; Blatt, R.; Catani, J.; Celi, A.; Cirac, J.I.; Dalmonte, M.; Fallani, L.; Jansen, K.; Lewenstein, M.; Montangero, S.; et al. Simulating Lattice Gauge Theories within Quantum Technologies. Eur. Phys. J. D 2020, 74, 165. [CrossRef]

25. Jaksch, D.; Bruder, C.; Cirac, J.I.; Gardiner, C.W.; Zoller, P. Cold Bosonic Atoms in Optical Lattices. Phys. Rev. Lett. 1998, 81,3108-3111. [CrossRef]

26. Tagliacozzo, L.; Celi, A.; Zamora, A.; Lewenstein, M. Optical Abelian Lattice Gauge Theories. Ann. Phys. 2013, 330, 160-191. [CrossRef]

27. Tagliacozzo, L.; Celi, A.; Orland, P.; Lewenstein, M. Simulations of non-Abelian gauge theories with optical lattices. Nat. Commun. 2013, 4, 2615. [CrossRef]

28. Zohar, E.; Cirac, J.I.; Reznik, B. Cold-Atom Quantum Simulator for SU(2) Yang-Mills Lattice Gauge Theory. Phys. Rev. Lett. 2013, 110, 125304. [CrossRef]

29. Zohar, E.; Cirac, J.I.; Reznik, B. Quantum simulations of gauge theories with ultracold atoms: local gauge invariance from angular momentum conservation. Phys. Rev. A 2013, 88, 023617. [CrossRef]

30. Zohar, E.; Cirac, J.I.; Reznik, B. Quantum Simulations of Lattice Gauge Theories using Ultracold Atoms in Optical Lattices. Rept. Prog. Phys. 2016, 79, 014401. [CrossRef]

31. Dasgupta, R.; Raychowdhury, I. Cold Atom Quantum Simulator for String and Hadron Dynamics in Non-Abelian Lattice Gauge Theory. arXiv 2020, arXiv:2009.13969.

32. Cirac, J.I.; Zoller, P. Quantum Computations with Cold Trapped Ions. Phys. Rev. Lett. 1995, 74, 4091-4094. [CrossRef]

33. Schindler, P.; Nigg, D.; Monz, T.; Barreiro, J.T.; Martinez, E.; Wang, S.X.; Quint, S.; Brandl, M.F.; Nebendahl, V.; Roos, C.F.; et al. A quantum information processor with trapped ions. New J. Phys. 2013, 15, 123012. [CrossRef]

34. Martinez, E.A.; Muschik, C.A.; Schindler, P.; Nigg, D.; Erhard, A.; Heyl, M.; Hauke, P.; Dalmonte, M.; Monz, T.; Zoller, P.; et al. Real-time dynamics of lattice gauge theories with a few-qubit quantum computer. Nature 2016, 534, 516-519. [CrossRef]

35. You, J.Q.; Nori, F. Superconducting Circuits and Quantum Information. Phys. Today 2005, 58, 42-47. [CrossRef]

36. Marcos, D.; Widmer, P.; Rico, E.; Hafezi, M.; Rabl, P.; Wiese, U.J.; Zoller, P. Two-dimensional Lattice Gauge Theories with Superconducting Quantum Circuits. Ann. Phys. 2014, 351, 634-654. [CrossRef]

37. Mezzacapo, A.; Rico, E.; Sabin, C.; Egusquiza, I.L.; Lamata, L.; Solano, E. Non-Abelian SU(2) Lattice Gauge Theories in Superconducting Circuits. Phys. Rev. Lett. 2015, 115, 240502. [CrossRef]

38. Alexandru, A.; Bedaque, P.F.; Lamm, H.; Lawrence, S. $\sigma$ Models on Quantum Computers. Phys. Rev. Lett. 2019, 123, 090501. [CrossRef] 
39. Singh, H.; Chandrasekharan, S. Qubit regularization of the $O(3)$ sigma model. Phys. Rev. D 2019, 100, 054505. [CrossRef]

40. Singh, H. Qubit $O(N)$ nonlinear sigma models. arXiv 2019, arXiv:1911.12353.

41. Bhattacharya, T.; Buser, A.J.; Chandrasekharan, S.; Gupta, R.; Singh, H. Qubit regularization of asymptotic freedom. Phys. Rev. Lett. 2021, 126, 172001. [CrossRef]

42. Zhou, J.; Singh, H.; Bhattacharya, T.; Chandrasekharan, S.; Gupta, R. Space-time symmetric qubit regularization of the asymptotically free two-dimensional O(4) model. arXiv 2021, arXiv:2111.13780.

43. Chandrasekharan, S.; Wiese, U.J. Quantum link models: A Discrete approach to gauge theories. Nucl. Phys. B 1997, $492,455-474$. [CrossRef]

44. Brower, R.; Chandrasekharan, S.; Wiese, U.J. QCD as a quantum link model. Phys. Rev. D 1999, 60, 094502. [CrossRef]

45. Brower, R.; Chandrasekharan, S.; Riederer, S.; Wiese, U.J. D theory: Field quantization by dimensional reduction of discrete variables. Nucl. Phys. B 2004, 693, 149-175. [CrossRef]

46. Banerjee, D.; Bögli, M.; Dalmonte, M.; Rico, E.; Stebler, P.; Wiese, U.J.; Zoller, P. Atomic Quantum Simulation of U(N) and SU(N) Non-Abelian Lattice Gauge Theories. Phys. Rev. Lett. 2013, 110, 125303. [CrossRef]

47. Wiese, U.J. Ultracold Quantum Gases and Lattice Systems: Quantum Simulation of Lattice Gauge Theories. Ann. Phys. 2013, 525, 777-796. [CrossRef]

48. Wiese, U.J. Towards Quantum Simulating QCD. Nucl. Phys. A 2014, 931, 246-256. [CrossRef]

49. Wiese, U.J. From Quantum Link Models to D-Theory: A Resource Efficient Framework for the Quantum Simulation and Computation of Gauge Theories. arXiv 2021, arXiv:2107.09335.

50. Bender, J.; Zohar, E.; Farace, A.; Cirac, J.I. Digital quantum simulation of lattice gauge theories in three spatial dimensions. New J. Phys. 2018, 20, 093001. [CrossRef]

51. Hackett, D.C.; Howe, K.; Hughes, C.; Jay, W.; Neil, E.T.; Simone, J.N. Digitizing Gauge Fields: Lattice Monte Carlo Results for Future Quantum Computers. Phys. Rev. A 2019, 99, 062341. [CrossRef]

52. Lamm, H.; Lawrence, S.; Yamauchi, Y. General Methods for Digital Quantum Simulation of Gauge Theories. Phys. Rev. D 2019, 100, 034518. [CrossRef]

53. Alexandru, A.; Bedaque, P.F.; Harmalkar, S.; Lamm, H.; Lawrence, S.; Warrington, N.C. Gluon Field Digitization for Quantum Computers. Phys. Rev. D 2019, 100, 114501. [CrossRef]

54. Preskill, J. Quantum Computing in the NISQ era and beyond. Quantum 2018, 2, 79. [CrossRef]

55. Byrnes, T.; Yamamoto, Y. Simulating lattice gauge theories on a quantum computer. Phys. Rev. A 2006, 73, 022328. [CrossRef]

56. Tagliacozzo, L.; Celi, A.; Lewenstein, M. Tensor Networks for Lattice Gauge Theories with continuous groups. Phys. Rev. X 2014, 4, 041024. [CrossRef]

57. Zohar, E.; Burrello, M. Formulation of lattice gauge theories for quantum simulations. Phys. Rev. D 2015, 91, 054506. [CrossRef]

58. Klco, N.; Stryker, J.R.; Savage, M.J. SU(2) non-Abelian gauge field theory in one dimension on digital quantum computers. Phys. Rev. D 2020, 101, 074512. [CrossRef]

59. Sandvik, A.W.; Scalapino, D.J. Order-disorder transition in a two-layer quantum antiferromagnet. Phys. Rev. Lett. 1994, 72, 2777-2780. [CrossRef]

60. Sachdev, S. Quantum Phase Transitions; Cambridge University Press: Cambridge, UK, 2000. [CrossRef]

61. Beard, B.; Pepe, M.; Riederer, S.; Wiese, U. Study of CP(N-1) theta-vacua by cluster-simulation of SU(N) quantum spin ladders. Phys. Rev. Lett. 2005, 94, 010603. [CrossRef]

62. Ciavarella, A.; Klco, N.; Savage, M.J. Trailhead for quantum simulation of SU(3) Yang-Mills lattice gauge theory in the local multiplet basis. Phys. Rev. D 2021, 103, 094501. [CrossRef]

63. Zache, T.V.; Van Damme, M.; Halimeh, J.C.; Hauke, P.; Banerjee, D. Achieving the continuum limit of quantum link lattice gauge theories on quantum devices. arXiv 2021, arXiv:2104.00025.

64. Meurice, Y.; Sakai, R.; Unmuth-Yockey, J. Tensor lattice field theory with applications to the renormalization group and quantum computing. arXiv 2020, arXiv:2010.06539.

65. The GAP Group. GAP_Groups, Algorithms, and Programming, Version 4.11.1. 2021. Available online: https://www.gapsystem.org (accessed on 3 September 2021).

66. Zhang, J.; Meurice, Y.; Tsai, S.W. Truncation effects in the charge representation of the O(2) model. Phys. Rev. B 2021, 103, 245137. [CrossRef]

67. Lang, S. Algebra; Graduate Texts in Mathematics; Springer: New York, NY, USA, 2005.

68. Ribet, K.A. On $\ell$-adic representations attached to modular forms. Invent. Math. 1975, 28, 245-275. [CrossRef]

69. Inonu, E.; Wigner, E.P. On the Contraction of groups and their represenations. Proc. Nat. Acad. Sci. USA 1953, 39, 510-524. [CrossRef]

70. Kogut, J.B.; Susskind, L. Hamiltonian Formulation of Wilson's Lattice Gauge Theories. Phys. Rev. D 1975, 11, 395-408. [CrossRef]

71. Harlow, D.; Ooguri, H. Symmetries in quantum field theory and quantum gravity. Commun. Math. Phys. 2021, 383, 1669-1804. [CrossRef]

72. Bronzan, J.B. Parametrization of SU(3). Phys. Rev. D 1988, 38, 1994. [CrossRef] 